\title{
Article \\ Highly-Efficient Sulfonated UiO-66(Zr) Optical Fiber for Rapid Detection of Trace Levels of $\mathrm{Pb}^{2+}$
}

\author{
Marziyeh Nazari ${ }^{1,2} \mathbb{D}^{\mathbb{D}}$, Abbas Amini ${ }^{3,4, * \mathbb{D}}$, Nathan T. Eden ${ }^{5} \mathbb{D}$, Mikel C. Duke ${ }^{2} \mathbb{D}$ and Chun Cheng ${ }^{6} \mathbb{D}$ \\ and Matthew R. Hill 5,7 (D)
}

1 Mathematics and Physics Department, School of Engineering, Australian College of Kuwait, Safat 13015, Kuwait; m.nazari@ack.edu.kw

2 Institute for Sustainable Industries and Livable Cities (ISILC), Victoria University, Melbourne, VIC 8001, Australia; mikel.duke@vu.edu.au

3 Mechanical Engineering Department, School of Engineering, Australian College of Kuwait, Safat 13015, Kuwait

4 Center for Infrastructure Engineering, Western Sydney University, Penrith, NSW 2751, Australia

5 Department of Chemical Engineering, Monash University, Clayton, VIC 3800, Australia; nathan.eden@monash.edu (N.T.E.); matthew.hill@csiro.au (M.R.H.)

6 Department of Materials Science and Engineering, Southern University of Science and Technology (SUSTech), Shenzhen 518055, China; chengc@sustech.edu.cn

7 CSIRO Manufacturing, Clayton, VIC 3168, Australia

* Correspondence: a.amini@ack.edu.kw

Citation: Nazari, M.; Amini, A.; Eden, N.T.; Duke, M.C.; Cheng, C.; Hill, M.R. Highly-Efficient Sulfonated UiO-66(Zr) Optical Fiber for Rapid Detection of Trace Levels of $\mathrm{Pb}^{2+}$. Int. J. Mol. Sci. 2021, 22, 6053. https:// doi.org/10.3390/ijms22116053

Academic Editor: Ana María Díez-Pascual

Received: 5 May 2021

Accepted: 28 May 2021

Published: 3 June 2021

Publisher's Note: MDPI stays neutral with regard to jurisdictional claims in published maps and institutional affiliations.

Copyright: (c) 2021 by the authors. Licensee MDPI, Basel, Switzerland. This article is an open access article distributed under the terms and conditions of the Creative Commons Attribution (CC BY) license (https:// creativecommons.org/licenses/by/ $4.0 /)$.

\begin{abstract}
Lead detection for biological environments, aqueous resources, and medicinal compounds, rely mainly on either utilizing bulky lab equipment such as ICP-OES or ready-made sensors, which are based on colorimetry with some limitations including selectivity and low interference. Remote, rapid and efficient detection of heavy metals in aqueous solutions at ppm and sub-ppm levels have faced significant challenges that requires novel compounds with such ability. Here, a UiO-66(Zr) metal-organic framework (MOF) functionalized with $\mathrm{SO}_{3} \mathrm{H}$ group $\left(\mathrm{SO}_{3} \mathrm{H}-\mathrm{UiO}-66(\mathrm{Zr})\right)$ is deposited on the end-face of an optical fiber to detect lead cations $\left(\mathrm{Pb}^{2+}\right)$ in water at 25.2, 43.5 and $64.0 \mathrm{ppm}$ levels. The $\mathrm{SO}_{3} \mathrm{H}-\mathrm{UiO}-66(\mathrm{Zr})$ system provides a Fabry-Perot sensor by which the lead ions are detected rapidly (milliseconds) at $25.2 \mathrm{ppm}$ aqueous solution reflecting in the wavelength shifts in interference spectrum. The proposed removal mechanism is based on the adsorption of $\left[\mathrm{Pb}\left(\mathrm{OH}_{2}\right)_{6}\right]^{2+}$ in water on $\mathrm{SO}_{3} \mathrm{H}-\mathrm{UiO}-66(\mathrm{Zr})$ due to a strong affinity between functionalized MOF and lead. This is the first work that advances a multi-purpose optical fiber-coated functional MOF as an on-site remote chemical sensor for rapid detection of lead cations at extremely low concentrations in an aqueous system.
\end{abstract}

Keywords: nano-bio detectors and sensors; nano-bio systems; aqueous quality; nanobiotechnology; optical fiber vesicle; sulfonated MOFs

\section{Introduction}

Lead $\left(\mathrm{Pb}^{2+}\right)$ and other small-scale substances (e.g., soot aerosol, ammonia, and arsenic) [1,2] are known as deadly widespread toxic pollutants in the environment at macroto nano-scale due to recent industrialization and agricultural activities [3,4]. A serious concern has raised for Canadian [5], the U.S. [6], and old European mega-cities [7] with high amounts of lead nano-substances found in drinking water which are originated from old pipes or chemical reactions occurred in corroded plumbing components [8].

According to American Academy of Family Physicians (AAFP), any level of detectable lead in human blood is abnormal [9]. Recurring exposure to low levels of $\mathrm{Pb}$ creates serious health issues for infants and children such as slow development and permanent intellectual disability $[10,11]$. In addition to various sources, lead can be taken up by fishes and other aquatic organisms from water accumulating in humans tissues after consumption [12], 
and then resulting in neurological [13], hematopoietic [14], musculoskeletal [15], cardiac function [16] and reproductive damages [17,18]. Despite the detrimental properties of lead to living objects, there is yet a significant gap to efficiently and abruptly detect and characterize lead at extremely small levels in bioactive compounds [19,20].

Methodologically, atomic absorption spectroscopy (AAS), atomic emission spectroscopy (AES), X-ray fluorescence (XRF), and inductively coupled plasma-optical emission spectrometry (ICP-OES) are the commonly used laboratory techniques for measuring lead contents in drinking water [21]. To use the above expensive and complicated equipment, water samples should be collected on-site, transported to a laboratory, and tested by trained professionals. This process for a large-scale determination of lead concentration is costly, time-consuming, and effortful. As yet, there are considerable efforts to develop sensors to allow discrete measurements of lead contents at-the-source for home-users. The existing detection mechanisms are based on colorimetry [22,23], biosensing [24], and electrochemical configurations [25], which, in addition to their low detection limits, have many other constraints. For instance, matrix interferences in the colorimetric method either disrupt the reaction between the reagent and the analyte or interfere the spectrometric light measurement [21]. In the biosensing method, more complex biological molecules, e.g., Daphnia magna, are needed with higher selectivity and less interference for reagents [26]. In the electrochemical sensing technique, lead-selective membranes are utilized on electrodes, where the response can be impacted by the interference from other ions presented in the water sample, effecting the solution ionic strength and potential drift [21]. Thus, an accessible, fast, sustainable, and efficient technology can fill this gap to detect $\mathrm{Pb}^{2+}$ in aqueous resources at low ppm and ppb levels [27].

So far, there are increasing interests in recent years to take advantage of advanced materials to adsorb $\mathrm{Pb}^{2+}$ nanoparticles at deficit concentrations from aqueous resources [28-33]. Metal-organic frameworks (MOFs) are highly porous 3D-materials made of metal ions linked with organic ligands. The size and shape of pores are affected by the coordination geometry of metals (e.g., tetrahedral, octahedral) that dictates the number of bounded ligands. Proper selection of metal ions and ligands can yield crystals with ultrahigh porosity as well as high thermal and chemical stability. Among MOFs, UiO-66(Zr) or $\mathrm{Zr}_{6} \mathrm{O}_{4}(\mathrm{OH})_{4}$ has a stable crystalline structure in water, introducing it as a promising candidate for sensing the aqueous contaminants and purification purposes [34]. Our recent studies have shown the favorable capability of UiO-66 for removing rhodamine-B [35], methyl viologen [36], and 4-aminopyridine [37] from water contents. The functionalization of $\mathrm{UiO}-66(\mathrm{Zr})$ with proper chemical groups is suggested to enhance its low affinity with $\mathrm{Pb}^{2+}$ ions.

In this work, a new setup is introduced via functionalization of $\mathrm{UiO}-66(\mathrm{Zr})$ with $\mathrm{SO}_{3} \mathrm{H}$ to capture $\mathrm{Pb}^{2+}$ at low ppm levels in aqueous environments. Due to the strong coordination of $\mathrm{Pb}^{2+}$ with $\mathrm{SO}_{3}^{-}$group, a small quantity of lead $(<0.5 \mathrm{ppm})$ was left in the solution. To create a remote sensing setup for rapid detection within the range of a few milliseconds, the functionalized MOF was coated at the end-face of an optical fiber (single-mode fiber, SMF-28) and used as an in-fiber Fabry-Perot interferometer (FPI) [38]. The changes to the MOF optical properties due to adsorption of $\mathrm{Pb}^{2+}$ were detected via wavelength shifts in the interference spectrum.

\section{Methods}

$\mathrm{SO}_{3} \mathrm{H}-\mathrm{UiO}-66(\mathrm{Zr})$ sensing element was synthesized through a growth solution proposed by Okoro et al. [24] with some modifications. Briefly, $\mathrm{ZrCl}_{4}(1.93 \mathrm{~g}, 8.3 \mathrm{mmol})$ and monosodium 2-sulfoterephthalate $\left(\mathrm{NaSO}_{3}-\mathrm{BDC}, 2.2 \mathrm{~g}, 8.2 \mathrm{mmol}\right)$ were dissolved under stirring with $\mathrm{N}, \mathrm{N}$-dimethylformamide (DMF, $100 \mathrm{~mL})$ and concentrated $\mathrm{HCl}(37 \%$, $1.3 \mathrm{~mL})$. Then, glacial acetic acid $(100 \%, 16.6 \mathrm{~mL}$; 35 equiv.) was added as a modulator. The mixture was continuously stirred for $2 \mathrm{~h}$ and left for $24 \mathrm{~h}$ at $120^{\circ} \mathrm{C}$ in a pre-heated oven. After naturally cooling down to room temperature, the $\mathrm{SO}_{3} \mathrm{H}-\mathrm{UiO}-66(\mathrm{Zr})$ nanoparticles were centrifuged at 20,000 rpm for $10 \mathrm{~min}$ and washed with fresh DMF (at least three times), then with pure methanol (at least three times), and kept under constant 
stirring with dichloromethane (DCM) overnight. They were dried for $5 \mathrm{~h}$ at $60{ }^{\circ} \mathrm{C}$ under reduced pressure.

The SMF-28 optical fiber was made of molten silica glass heated up to $2200{ }^{\circ} \mathrm{C}$ and drawn into tubes with varied diameters. In this work, fibers with a cladding diameter of $125 \pm 0.7 \mu \mathrm{m}$ and a core diameter of $8.2 \mu \mathrm{m}$ were utilized. Before coating, the optical fiber made of $\mathrm{SiO}_{2}$ glass was treated with hydroxyl $(\mathrm{OH})$ functional groups. A piranha solution was prepared from a 3:1 mixture of sulfuric acid (98\%) and hydrogen peroxide (30\%), into which optical fibers were cleaved at a right angle and incubated for $30 \mathrm{~min}$. To grow the MOF sensing element on the exposed surface of the optical fibers, the $\mathrm{OH}$-functionalized optical fibers were placed in an untreated precursor solution of $\mathrm{SO}_{3} \mathrm{H}-\mathrm{UiO}-66(\mathrm{Zr})$, heated at $120^{\circ} \mathrm{C}$ for $24 \mathrm{~h}$. After this procedure, the fibers were gently washed with DMF, methanol and DCM, to remove unreacted reagents.

Fabry-Perot interferometry (FPI) was used as the optical detection method. When the light is propagated down to the core of fiber, it interacts with the sensing element. The element is in contact with lead-contaminated water, and by capturing the $\mathrm{Pb}^{2+}$, its optical thickness and refractive index change. The reflected light sends this information to the detector, where a custom-written software (MATLAB [39]) processes it [35-37]. The resultant FPI spectrum (interferograms) is obtained in correlation with the lead concentration in distilled (DI) water.

To test the lead uptake capacity of as-synthesized MOF, different amounts of $\mathrm{SO}_{3} \mathrm{H}-$ UiO-66( $\mathrm{Zr})(10,15,20$ and $25 \mathrm{mg})$ were separately added to $5 \mathrm{~mL}$ diluted solution with $25.2(0.12 \mathrm{mM}), 43.5(0.21 \mathrm{mM})$ and $64.0(0.31 \mathrm{mM}) \mathrm{ppm}$ lead under constant stirring for 10,30 and $60 \mathrm{~min}$ (impregnation). Before taking a sample (aliquot) from the middle of the vial, the solid was separated by centrifuging at 20,000 rpm for $5 \mathrm{~min}$, then resting for $4 \mathrm{~h}$ at room temperature. The adsorption mechanism of $\mathrm{Pb}^{2+}$ onto $\mathrm{SO}_{3} \mathrm{H}-\mathrm{UiO}-66(\mathrm{Zr})$ was characterized by $\mathrm{X}$-ray diffraction $(\mathrm{XRD}), \mathrm{N}_{2}$ gas porosimetry, and Fourier-transform infrared spectroscopy (FT-IR) methods (Appendix A).

\section{Results and Discussion}

\section{1. $\mathrm{Pb}^{2+}$ Uptake by $\mathrm{SO}_{3} \mathrm{H}-\mathrm{UiO}-66(\mathrm{Zr})$ Powder}

Figure 1a displays the $\mathrm{XRD}$ pattern of $\mathrm{SO}_{3} \mathrm{H}-\mathrm{UiO}-66(\mathrm{Zr})$ and $\mathrm{SO}_{3} \mathrm{~Pb}-\mathrm{UiO}-66(\mathrm{Zr})$ powder. A change in the XRD pattern of the amorphous $\mathrm{SO}_{3} \mathrm{H}-\mathrm{UiO}-66(\mathrm{Zr})$ sample occurrs by appearing the additional peaks in the $\mathrm{SO}_{3} \mathrm{~Pb}-\mathrm{UiO}-66(\mathrm{Zr})$ sample (prescribed to $\mathrm{PbSO}_{4}$ post-adsorption through peak matching in crystallographic database (Figure A1)). This suggests the formation of a material that matches with the crystallographic geometry of $\mathrm{PbSO}_{4}$. The cleavage energy of the sulfonate group is prohibitively high under the adsorption conditions ( $\mathrm{pH}=5.6$, room temperature/pressure, and in aqueous environment), and $\mathrm{Pb}\left(\mathrm{NO}_{3}\right)_{2}$ is soluble in water with different crystallographic geometries. The $\mathrm{pH}$ was achieved by atmospheric carbon dioxide dissolving into the DI water as a natural process. To avoid addition of interferent compounds to the water/ $\mathrm{Pb}$ context, the $\mathrm{pH}$ was not regulated. Therefore, we conclude that $\mathrm{Pb}^{2+}$ cation is absorbed onto the sulfonate groups of $\mathrm{SO}_{3} \mathrm{H}-\mathrm{UiO}-66(\mathrm{Zr})$ structure where the tetrahedral $\mathrm{R}^{-\mathrm{SO}_{3}}$ of the 2-sulfoterephthalate linker replaces the tetrahedral $\mathrm{SO}_{4}$ of $\mathrm{PbSO}_{4}$.

Standard $\mathrm{N}_{2}$ gas porosimetry measurements confirm the uptake of lead within $\mathrm{SO}_{3} \mathrm{H}$ UiO-66(Zr), where the Brunauer-Emmett-Teller (BET) surface area decreases from $491 \mathrm{~m}^{2} / \mathrm{g}$ to $12 \mathrm{~m}^{2} / \mathrm{g}$ upon the lead uptake. Figure $1 \mathrm{~b}$ shows the pore size distribution of $\mathrm{SO}_{3} \mathrm{H}-\mathrm{UiO}-$ $66(\mathrm{Zr})$ before/after the lead uptake; this is associated with the additional mass of $\mathrm{Pb}^{2+}$ incorporated into the compound as well as the reduction of internal pore volume. The adsorption/desorption isotherms of $\mathrm{SO}_{3} \mathrm{H}-\mathrm{UiO}-66(\mathrm{Zr})$ before/after the lead uptake are shown in Figure 1c. 


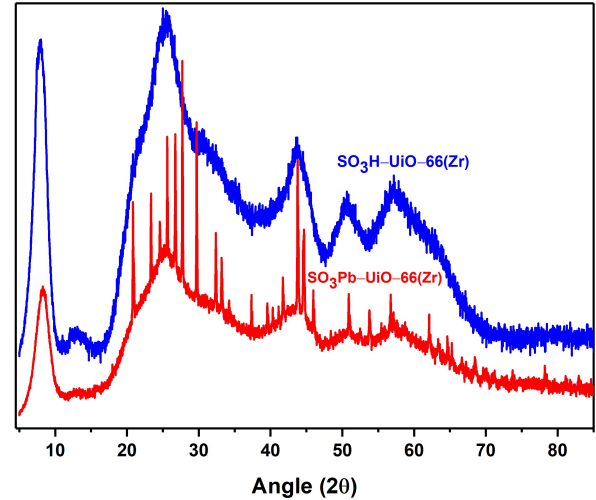

(a)

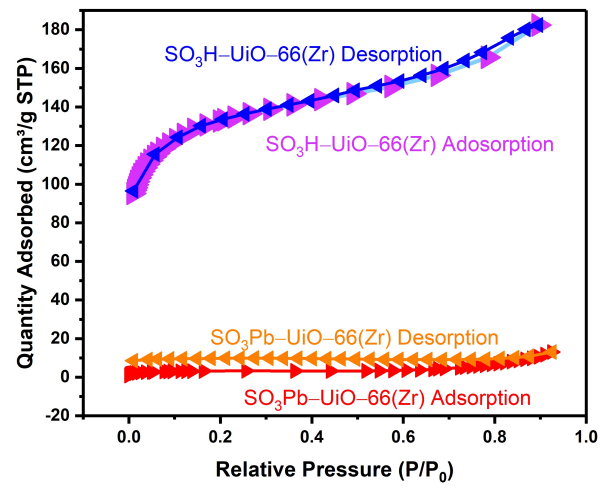

(c)

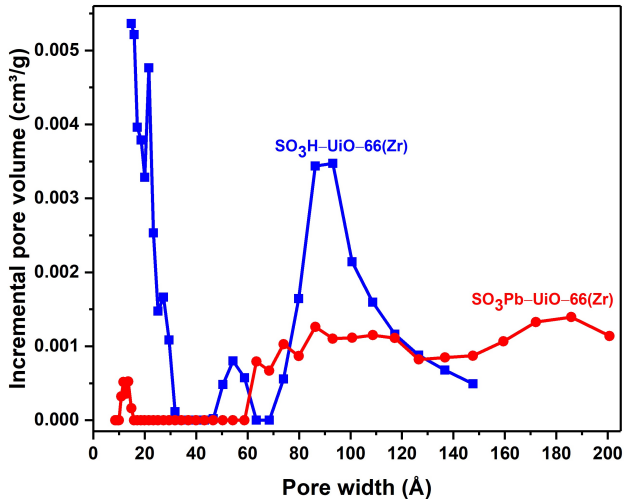

(b)

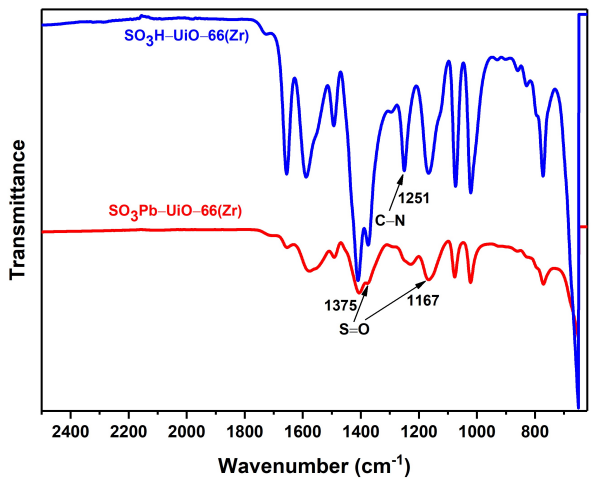

(d)

Figure 1. (a) XRD curves, (b) Pore size distribution, (c) $\mathrm{N}_{2}$ adsorption/desorption isotherms, and (d) FT-IR spectra of $\mathrm{SO}_{3} \mathrm{H}-\mathrm{UiO}-66(\mathrm{Zr})$ and $\mathrm{SO}_{3} \mathrm{~Pb}-\mathrm{UiO}-66(\mathrm{Zr})$ powder.

Upon the uptake of $\mathrm{Pb}^{2+}$, significant changes in the observed transmittance reflect the change of dipole moment due to the adsorbed $\mathrm{Pb}^{2+}$. Unchanged peak positions associated with the $\mathrm{UiO}-66(\mathrm{Zr})$ framework indicate that the structure does not undergo significant changes while the crystallinity increases (Figure 1d). For instance, the characteristic $\mathrm{S}=\mathrm{O}$ stretching at $1375 \mathrm{~cm}^{-1}$ and $1167 \mathrm{~cm}^{-1}$ become weaker in the $\mathrm{SO}_{3} \mathrm{~Pb}-\mathrm{UiO}-66(\mathrm{Zr})$ sample, while $\mathrm{C}-\mathrm{H}$ stretching peak at $1251 \mathrm{~cm}^{-1}$ has a very low intensity. Defects in $\mathrm{SO}_{3} \mathrm{H}-\mathrm{UiO}-$ $66(\mathrm{Zr})$ reduces the density of functional groups that interact with the laser. The reduced transmittance is seen in the figure in the gesture of smaller peaks.

\section{2. $\mathrm{Pb}^{2+}$ Uptake of $\mathrm{SO}_{3} \mathrm{H}-\mathrm{UiO}-66(\mathrm{Zr})$ Powder}

Inductively coupled plasma-optical emission spectrometry (ICP-OES) was conducted to determine the trace level of lead in the aliquots after its impregnation. By nonlinear regression modelling, the equilibrium/optimized level of lead uptake capacity of assynthesized MOF was determined as $33.7 \mathrm{mg}$ with a maximum $94 \%$ uptake (r-squared fit of $99.7 \%)$.

Langmuir and Freundlich models are used to investigate the adsorbent/adsorbate interaction in MOFs. Langmuir isotherm assumes that in the uptake process, the adsorbent places itself as a monolayer on the surface of the material. This model can be linearly expressed in the form of Equation (1):

$$
C_{e} / q_{e}=K_{L} / q_{m}+C_{e} / q_{m}
$$

where $C_{e}$ is the equilibrium solution concentration (mg/L or ppm), $q_{e}$ is the amount of $\mathrm{Pb}^{2+}$ at the equilibrium $(\mathrm{mg} / \mathrm{g}), K_{L}$ is the Langmuir adsorption constant related to the energy of adsorption, and $q_{m}$ is the maximum adsorption capacity of the MOF $(\mathrm{mg} / \mathrm{g})$. A plot of $C_{e} / q_{e}$ (y-axis) vs. $C_{e}$ (x-axis) allows the calculation of $q_{m}$ and $K_{L}$ parameters (Figure 2a). 
Another model, empirical Freundlich isotherm, assumes that the distribution of active sites in the MOF is homogeneous and is linearly expressed in the form of Equation (2):

$$
\ln q_{e}=\ln K_{f}+\ln C_{e} / n
$$

here, $C_{e}$ is the equilibrium solution concentration (mg/L or ppm), $q_{e}$ is the amount of $\mathrm{Pb}^{2+}$ at the equilibrium $(\mathrm{mg} / \mathrm{g}), K_{f}$ is the Freundlich adsorption constant, and $n$ is an empirical value. A plot of $\ln q_{e}$ (y-axis) vs. $\ln C_{e}$ (x-axis) determines the magnitudes of $K_{f}$ and $n$ (Figure 2b).

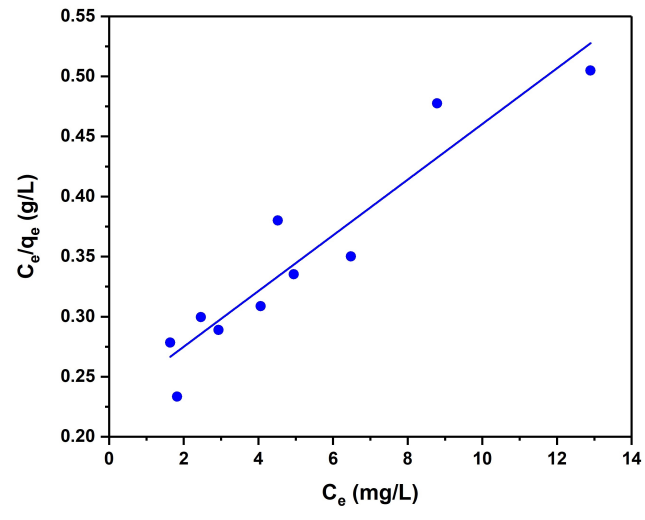

(a)

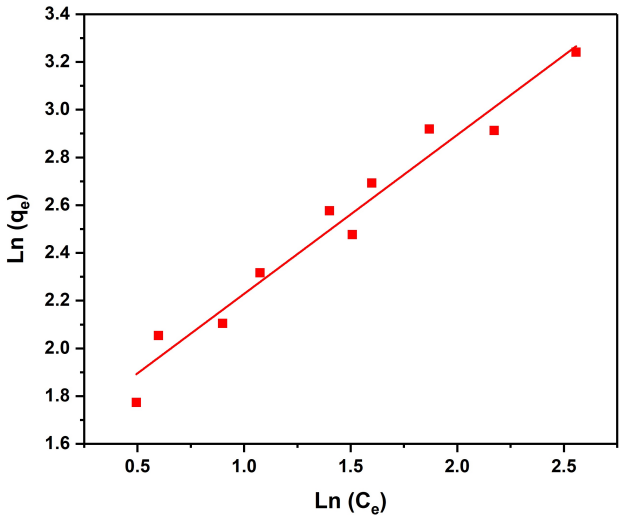

(b)

Figure 2. Adsorption isotherms fitted by linearized form of (a) Langmuir and (b) Freundlich model for adsorbed $\mathrm{Pb}^{2+}$ by $\mathrm{SO}_{3} \mathrm{H}-\mathrm{UiO}-66(\mathrm{Zr})$.

Regression analysis of the data, the average values of $\mathrm{K}_{f}, \mathrm{~K}_{L}, q_{m}$ and $n$ are shown in Table 1. In this study, the Freundlich model shows a better predictor than the Langmuir model with slightly higher $\mathrm{R}^{2}$, suggesting that the uptake of lead cations occurrs mainly and homogeneously throughout the entire of MOF framework. Moreover, the calculated average $n$ value for the adsorption of $\mathrm{Pb}^{2+}$ is 1.66 , showing a good efficiency of $\mathrm{SO}_{3} \mathrm{~Pb}$ UiO-66(Zr) for the lead adsorption $[40,41]$.

Table 1. Langmuir and Freundlich isotherm constants for the uptake of lead (II) cation at room temperature.

\begin{tabular}{cccccc}
\hline \multicolumn{3}{c}{ Langmuir Constants } & \multicolumn{3}{c}{ Freundlich Constants } \\
\hline $\boldsymbol{q}_{m}(\mathbf{m g} / \mathrm{g})$ & $K_{L}(\mathbf{m g} / \mathrm{L})$ & $\mathbf{R}^{2}$ & $K_{f}$ & $n$ & $\mathbf{R}^{2}$ \\
\hline 32.77 & 6.07 & 0.95 & 5.51 & 1.66 & 0.99 \\
\hline
\end{tabular}

To determine whether a high degree of crystallinity affected the lead uptake, the untreated precursor solution of $\mathrm{SO}_{3} \mathrm{H}-\mathrm{UiO}-66(\mathrm{Zr})$ was heated at $120{ }^{\circ} \mathrm{C}$ for $48 \mathrm{~h}$ (rather $24 \mathrm{~h}$ ); a highly crystallized as-synthesized MOF was achieved. As seen in the XRD patterns (Figure A2b), more defined Bragg peaks were observed for the MOF heated for $48 \mathrm{~h}$ in comparison to the one heated for $24 \mathrm{~h}$. Based on the ICP-OES results, $10 \mathrm{mg}$ of this highly formed MOF led to $<0.5 \mathrm{ppm}(2.4 \mu \mathrm{M})$ left-over lead in the initial $25.2 \mathrm{ppm}$ solution (99.99\% uptake) for all impregnation ranges (10, 30 and $60 \mathrm{~min})$. In contrast, non-functionalized UiO-66(Zr) MOF reduced the lead content from $25.2 \mathrm{ppm}$ to $23.1 \mathrm{ppm}$ after 60 min constant stirring.

\subsection{Optical Fiber Sensing}

After successfully validating the impregnation of lead within $\mathrm{SO}_{3} \mathrm{H}-\mathrm{UiO}-66(\mathrm{Zr})$, the coated element at the tip of optical fiber was utilized as a chemical sensor for the rapid (a few milliseconds) detection of lead in DI water. Figure 3a illustrates the SEM image of a deposited sensing element of sulfonated $\mathrm{SO}_{3} \mathrm{H}-\mathrm{UiO}-66(\mathrm{Zr})$ at the tip of optical fiber 
adjacent to a bare optical fiber in Figure $3 \mathrm{~b}$. The interference of two reflected beams was recorded using an optical spectrum analyzer (OSA, Ando Japan, AQ6317B, 600-1750 nm). An Agilent 83438A Erbium ASE (Agilent Technologies, Santa Rosa, CA, USA) was used as the light source with the wavelength range of $1500-1600 \mathrm{~nm}$. The OSA was set on a continuous scan mode to record the interference signals every $5 \mathrm{~s}$ with a high sensitivity and $0.1 \mathrm{~nm}$ resolution.

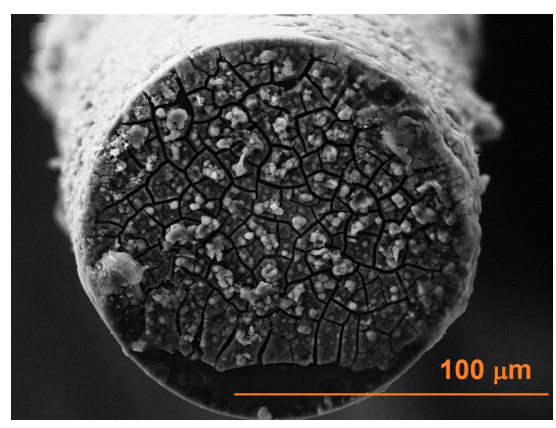

(a)

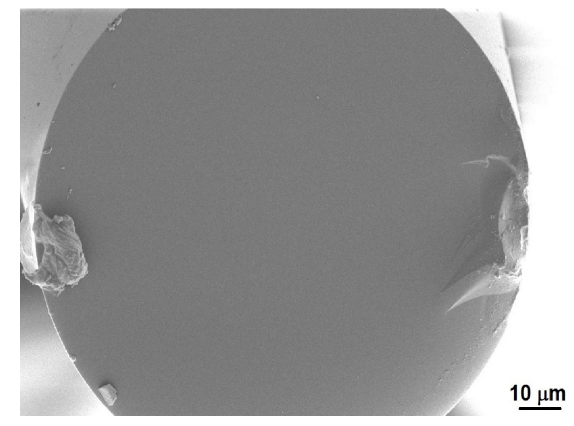

(b)

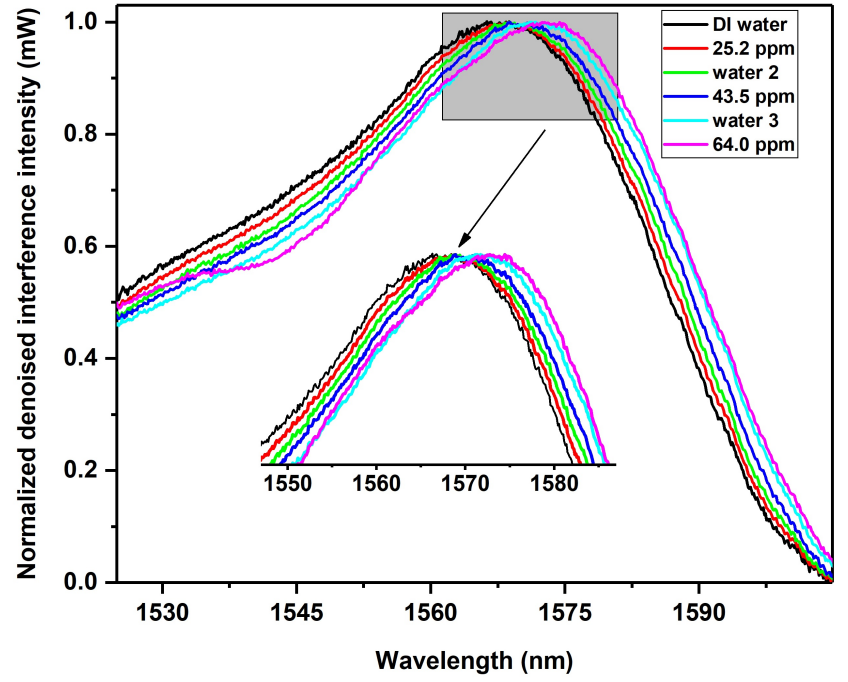

(c)

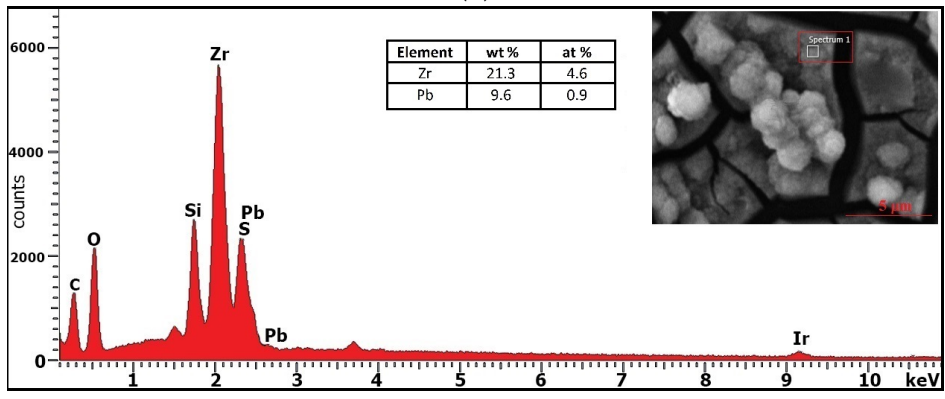

(d)

Figure 3. SEM images of (a) $\mathrm{SO}_{3} \mathrm{H}-\mathrm{UiO}-66(\mathrm{Zr})$ optical fiber sensing element, and (b) bare optical fiber, (c) Interferograms in correlation with a lead concentration in DI water, and (d) EDX spectra of $\mathrm{SO}_{3} \mathrm{H}-\mathrm{UiO}-66(\mathrm{Zr})$ optical fiber sensing element after the lead uptake.

The average of all signals (100 trials) for each concentration was used as the final interference signal for a specific concentration. The fast response of the sensing element towards lead uptake was withdrawn by comparing the shape of signals at different sweeps. Figure $3 \mathrm{c}$ shows the spectral positions of the interferogram peak (normalized denoised interference intensity vs. wavelength) generated by $\mathrm{SO}_{3} \mathrm{H}-\mathrm{UiO}-66(\mathrm{Zr})$ sensing element at different lead concentrations. Briefly, the sensor was first placed in DI water for $5 \mathrm{~min}$ to 
observe the pattern of sensing element upon the exposure to DI water. The same procedure was repeated after immersing the sensor in different lead solutions ("water 2" and "water 3 " with $25.2 \mathrm{ppm}$ and $43.5 \mathrm{ppm}$ lead contents, respectively). Upon the introduction of lead nitrate solutions, the position of interferogram peak shifted to longer wavelengths. This indicated an increase in the lead adsorption by $\mathrm{SO}_{3} \mathrm{H}-\mathrm{UiO}-66(\mathrm{Zr})$ sensing element and, thus, the optical thickness.

Energy-Dispersive X-ray (EDX) analysis was performed on the optical fiber sensing element after the lead uptake process, in order to confirm the attachment of lead with $\mathrm{SO}_{3} \mathrm{H}-\mathrm{UiO}-66(\mathrm{Zr})$. As shown in Figure 3d, despite some detection difficulty due to the similarity of the energy of $\mathrm{S}-\mathrm{K} \alpha$ and $\mathrm{Pb}-\mathrm{M} \alpha(2.309 \mathrm{vs.} 2.342 \mathrm{keV}$, respectively), zirconium ( $\mathrm{Zr}$ ) (as the main constituent of $\mathrm{Zr}$-based $\mathrm{MOF} \mathrm{SO}_{3} \mathrm{H}-\mathrm{UiO}-66$ ) had $21.3 \%$ weight while $\mathrm{Pb}$ possessed $9.6 \%$ level in the samples with higher $\mathrm{ppm}$. The presence of sulfur (S) confirmed the $\mathrm{SO}_{3} \mathrm{H}-\mathrm{UiO}-66(\mathrm{Zr})$ structure.

As the reference for lead level in drinking water is $5 \mu \mathrm{g} / \mathrm{L}$ (5 ppb) [42], precision evaluation studies should be conducted to further overcome the detection limit. Nevertheless, optical fibers and $\mathrm{SO}_{3} \mathrm{H}-\mathrm{UiO}-66(\mathrm{Zr})$ are stable within the range of $\mathrm{pH} 2-9[43,44]$. Yet, the remaining challenge is to consider the sensor in very harsh environments for prolonged periods of time where the wastewater might be extremely acidic or basic. Although the optical fiber sensors were not designed explicitly for this purpose, there is an opportunity to trigger the release of the guest species $\left(\mathrm{Pb}^{2+}\right)$ using light at a specific wavelength [45]. The $\mathrm{pH}$ change [46], ligand exchange [47], and ethanol regeneration solvent [48] have been proposed to recycle MOFs for further usages.

\subsection{Mechanism of $\mathrm{Pb}^{2+}$ Adsorption}

Figure 4 shows the chemistry mechanism where the sensing material coordinates with $\mathrm{Pb}^{2+}$ ions via $n$ sulfonate groups, $n \leq 6$.

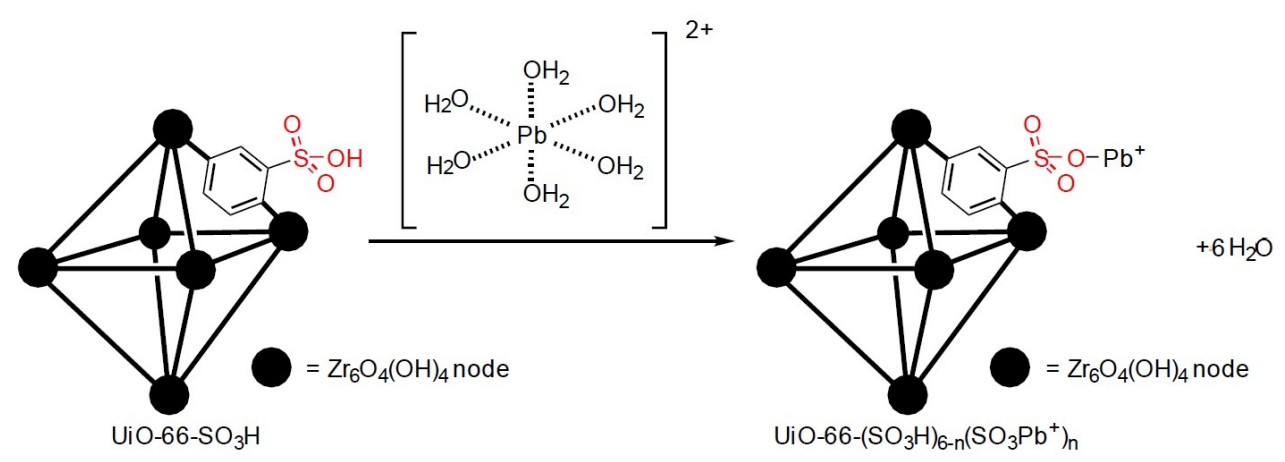

Figure 4. Diagram of adsorption of $\left[\mathrm{Pb}\left(\mathrm{OH}_{2}\right)_{6}\right]^{2+}$ on $\mathrm{SO}_{3} \mathrm{H}-\mathrm{UiO}-66(\mathrm{Zr})$ in water

As per our data, $\mathrm{Pb}$ adsorption occurrs in a $\mathrm{S}-\mathrm{O}-\mathrm{Pb}^{+}$bonding manner. There are 10 lone pairs of electrons present in the oxygens of sulfonic acid group, where the tetrahedral $\mathrm{R}-\mathrm{SO}_{3}$ geometry would prohibit the direct $\mathrm{S}-\mathrm{Pb}$ bonding. In fact, the presence of a crystalline phase identical to $\mathrm{Pb}\left(\mathrm{SO}_{4}\right)$ is due to the coordination of $\mathrm{Pb}$ to $\mathrm{SO}_{3}^{-}$group with the $\mathrm{O}$-carbon of 2-sulfonoterepthalic acid replacing one $\mathrm{O}$ in $\mathrm{Pb}\left(\mathrm{SO}_{4}\right)$. The rapid uptake of $\mathrm{Pb}$, shown from both the batch adsorption measurements and the optical fiber results, leads to both weak coordination and strong electrostatic attraction between $\mathrm{Pb}$ and $\mathrm{R}-\mathrm{O}^{-}$. The speciation of $\mathrm{Pb}\left(\mathrm{NO}_{3}\right)_{2}$ in aqueous solutions at the native $\mathrm{pH}$ of $\sim 5$ is mainly $\mathrm{Pb}^{2+}$, that exists within a solvation shell of approximately $6 \mathrm{H}_{2} \mathrm{O}$ molecules. The adsorptions would, therefore, have the following predominant reactions [49]:

$$
\begin{gathered}
R-\mathrm{SO}_{2} \mathrm{OH}_{(s)}+\mathrm{Pb}_{(a q)}^{2+} \longrightarrow \mathrm{R}-\mathrm{SO}_{3}^{-}+\mathrm{Pb}_{(a q)}^{2+}+\mathrm{H}_{3} \mathrm{O}_{(a q)}^{+} \longrightarrow \mathrm{R}-\mathrm{SO}_{2} \mathrm{O}-\mathrm{Pb}_{s}^{+} \\
R-\mathrm{SO}_{2} \mathrm{OH}_{(s)}+\mathrm{Pb}_{(a q)}^{2+} \longrightarrow R-\mathrm{SO}_{3}^{-}+\mathrm{Pb}_{(a q)}^{2+}+\mathrm{H}_{3} \mathrm{O}_{(a q)}^{+} \longrightarrow R-\mathrm{SO}_{3}^{-} \ldots \mathrm{Pb}_{s}^{2+}
\end{gathered}
$$


Equation (3) demonstrates an electrostatic attraction to a singular sulfonate $O$, while Equation (4) presents the coordination between the delocalized sulfonate $\mathrm{e}^{-}$and $\mathrm{Pb}^{2+}$. These are shown as a three-step process. The immersion of the MOF into water dissociates the sulfonic acid $\mathrm{H}^{+}$rapidly, after which lead is attracted to the sulfonate groups of $\mathrm{SO}_{3} \mathrm{H}-$ UiO-66( $\mathrm{Zr})$. Due to the rapid uptake, it is not expected that $\mathrm{Pb}(\mathrm{O} . \mathrm{H} .)^{-}$is adsorbed onto the sulfonates, as its fractional abundance at $\mathrm{pH} 5-6$ is low relative to the adsorption phenomenon [50].

\section{Conclusions}

The rapid detection of $\mathrm{Pb}^{2+}$ by MOF-coated optical fibers was introduced for the first time. A solvothermal synthesized sulfonic acid functionalized $\mathrm{MOF}, \mathrm{SO}_{3} \mathrm{H}-\mathrm{UiO}-$ $66(\mathrm{Zr})$, demonstrated the rapid uptake of lead from $\mathrm{Pb}\left(\mathrm{NO}_{3}\right)_{2}$ solution. The analysis of ICP-OES intensities showed the uptake capacity of $\mathrm{SO}_{3} \mathrm{H}-\mathrm{UiO}-66(\mathrm{Zr})$ as $32.77 \mathrm{mg} / \mathrm{g}$ $\left(\mathrm{R}^{2}=0.997\right)$. This MOF was grown on an OH-functionalized SMF-28 conventional singlemode optical fiber that acted as a detector for $\mathrm{Pb}^{2+}$ at the ppm-level concentrations. Spectral interferograms indicated the detection of $\mathrm{Pb}^{2+}$ down to $25.2 \mathrm{ppm}$. Such a MOF optical fiber can be implemented as a device for simple/abrupt on-site detection of aqueous $\mathrm{Pb}^{2+}$ or possibly other ions at ppm or sub-ppm levels. We envisage that this type of sensor would offer a novel and more effective composite to harvest heavy metals from contaminated water for clean water supply.

Further studies are currently undergoing to investigate the interference effects of a matrix containing more than one cation and anion in water, as other transition metal (II) ions may give a 'false' $\mathrm{Pb}^{2+}$ detection reading.

Author Contributions: All authors contributed equally. All authors have read and agreed to the published version of the manuscript.

Funding: The Kuwait Foundation for the Advancement of Sciences (KFAS) is acknowledged for funding No. PN18-15EC-01.

Institutional Review Board Statement: Not applicable.

Informed Consent Statement: Not applicable.

Data Availability Statement: The data presented in this study are available on request from the corresponding author.

Acknowledgments: The authors would like to express their gratitude towards Kristina Konstas, Muhammad M. Sadiq, Farnaz Zadehahmadi, Stephen F. Collins, Horace L. King, and Fairuza Faiz for their great assistance.

Conflicts of Interest: The authors declare no competing interests.

\section{Appendix A}

Appendix A.1. Sample Preparation and Analysis Conditions for X-ray Diffraction (XRD) Characterization

The sample was dry ground in a boron carbide mortar and pestle before being loaded into a low volume Si zero background sample holder. A "Bruker D8 Advance A25" X-ray Diffractometer operating under $\mathrm{CuK} \alpha$ radiation $(40 \mathrm{kV}, 40 \mathrm{~mA})$ and equipped with a Lynx Eye XE-T detector was employed to obtain the XRD patterns. The sample was scanned over the $2 \theta$ range of $5^{\circ}$ to $85^{\circ}$ at a step size of $0.02^{\circ}$ and a count time of $1.6 \mathrm{~s}$ per step and spun at 15 RPM during the data collection.

Analyses were performed on the collected XRD data using the Bruker XRD search match program EVA ${ }^{\mathrm{TM}} \mathrm{v} 5$. Crystalline phases were identified using the ICDD-JCPDS powder diffraction database. Pawley analyses were performed on the data using the Bruker TOPAS ${ }^{\mathrm{TM}} \mathrm{v} 6$ program to determine lattice parameters and crystallite size (Table A1). Background signal was described using a combination of Chebyshev polynomial linear interpolation function and $1 / x$ function. Cell parameters, vertical sample displacement, 
full peak width at half maximum, and peak scale factor were all refined. Error ranges were calculated based on three estimated standard deviations as calculated by TOPAS. A diffractogram pattern of $\mathrm{SO}_{3} \mathrm{~Pb}-\mathrm{UiO}-66(\mathrm{Zr})$ at $2 \theta$ region $\left(5-65^{\circ}\right)$ superimposed over the simulated diffractograms of $\mathrm{UiO}-66$ and $\mathrm{PbSO}_{4}$ anglesite is shown in Figure A1.

Table A1. Phase summary of $\mathrm{SO}_{3} \mathrm{H}-\mathrm{UiO}-66(\mathrm{Zr})$.

\begin{tabular}{|c|c|c|c|}
\hline & Lattice Parameter & Crystallite Size & Phase \\
\hline $24 \mathrm{~h}$ & NP (amorphous) & NP (amorphous) & UiO-66, except the $\left(\begin{array}{ll}0 & 2 \\
2\end{array}\right)$ peak at $\sim 12^{\circ} 2 \theta$ \\
\hline $48 \mathrm{~h}$ & $20.678 \pm 0.001 \AA$ & $170 \pm 11 \mathrm{~nm}$ & UiO-66, except the $\left(\begin{array}{lll}0 & 2 & 2\end{array}\right)$ peak at $\sim 12^{\circ} 2 \theta$ \\
\hline
\end{tabular}

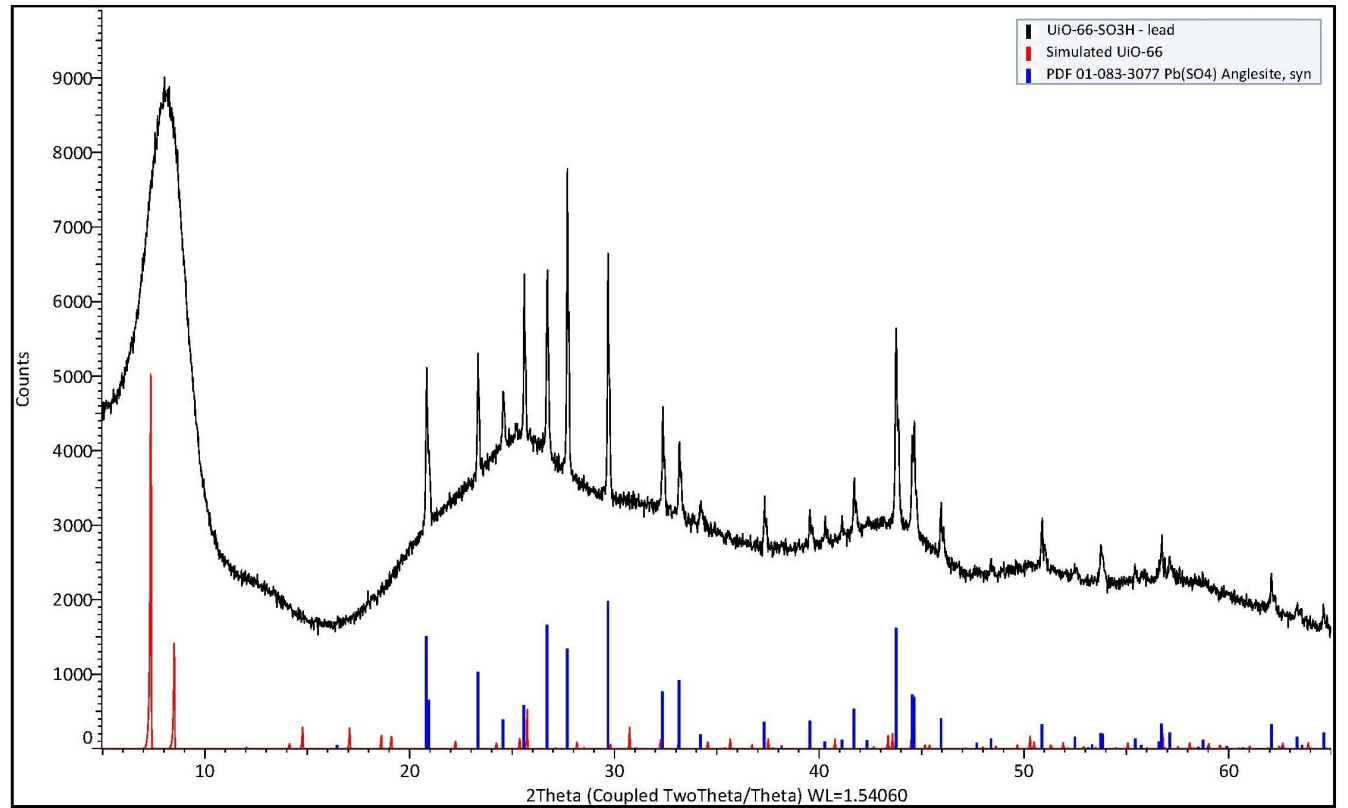

Figure A1. Diffractogram pattern of $\mathrm{SO}_{3} \mathrm{~Pb}-\mathrm{UiO}-66(\mathrm{Zr})$ at $2 \theta$ region $\left(5^{\circ}-65^{\circ}\right)$, superimposed over the simulated diffractograms of $\mathrm{UiO}-66$ and $\mathrm{PbSO}_{4}$ anglesite.

\section{Appendix A.2. $\mathrm{N}_{2}$ Gas Porosimetry}

Gas adsorption isotherms were measured for pressure within the range 0-120 $\mathrm{kPa}$ by a volumetric approach using a Micrometrics ASAP 2420 instrument. All samples were transferred to pre-dried and weighted analysis tubes and sealed with Transcal stoppers. They were evacuated and activated under a dynamic vacuum at $10^{-6}$ Torr at $140{ }^{\circ} \mathrm{C}$ for $8 \mathrm{~h}$. Ultra-high purity $\mathrm{N}_{2}$ was used for the experiments. $\mathrm{N}_{2}$ adsorption and desorption measurements were conducted at $77 \mathrm{~K}$. Surface area measurements were performed on $\mathrm{N}_{2}$ isotherms at $77 \mathrm{~K}$ using the Brunauer-Emmer-Teller (BET) model with increasing adsorption values of 0.005 to 0.2 .

\section{Appendix A.3. Inductively Coupled Plasma-Optical Emission Spectrometry (Icp-Oes) Analysis Method}

The samples were analyzed on an as-received basis by acidifying to $5(\mathrm{wt}) \% \mathrm{HNO}_{3}$. The solutions were then analyzed by using Varian 730-ES axial ICP-OES. Certified multi-element solutions were used to check the accuracy of the calibration standards and the method. 
Appendix A.4. Characterization of $\mathrm{So}_{3} \mathrm{H}-U$ Uio-66(Zr) (48 H Heating)

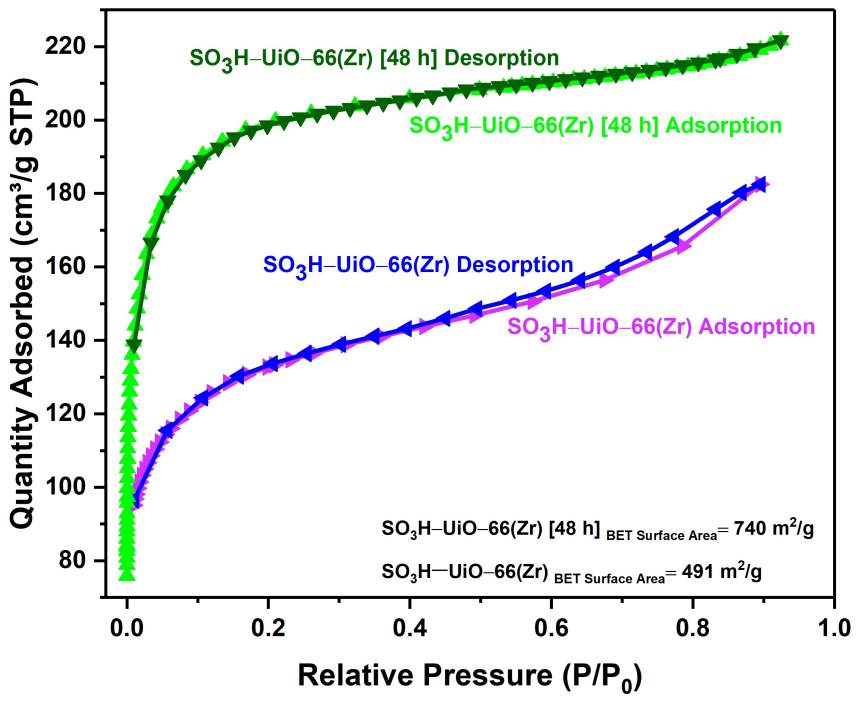

(a)

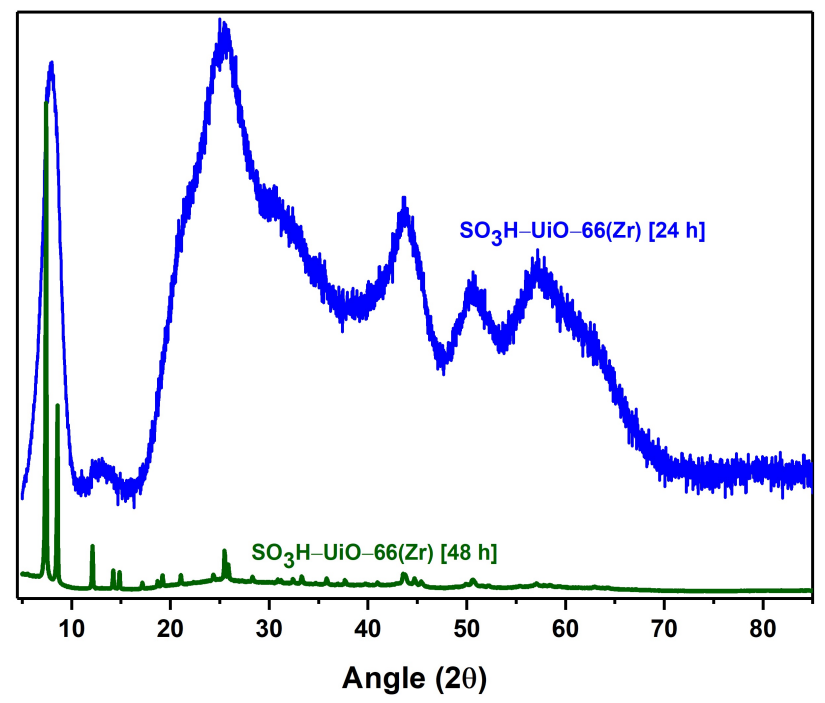

(b)

Figure A2. (a) $\mathrm{N}_{2}$ adsorption/desorption isotherms and (b) XRD curves of $\mathrm{SO}_{3} \mathrm{H}-\mathrm{UiO}-66(\mathrm{Zr})[24 \mathrm{~h}]$ and $\mathrm{SO}_{3} \mathrm{H}-\mathrm{UiO}-66(\mathrm{Zr})$ [48 h] powder.

\section{Appendix A.5. Optical Methodology}

The interference signal was processed in MATLAB [39] using a special two-stage signal processing algorithm. The first part of the algorithm used Daubechies Wave Transform (DWT) to remove noise from the spectrum. Then, the second part of the algorithm performed frequency domain analysis (fast Fourier transform (FFT)) to determine the response of the $\mathrm{SO}_{3} \mathrm{H}-\mathrm{UiO}-66(\mathrm{Zr})$ to lead concentration in DI water. Table A2 shows the raw data (Normalized denoised interference intensity (NDII) vs. Wavelength) obtained form one sensor after signal processing.

Table A2. Normalized denoised interference intensity (NDII).

\begin{tabular}{ccccccc}
\hline Wavelength & NDII & NDII & NDII & NDII & NDII & NDII \\
\hline $\mathbf{n m}$ & $\mathbf{m W}$ & $\mathbf{m W}$ & $\mathbf{m W}$ & $\mathbf{m W}$ & $\mathbf{m W}$ & $\mathbf{m W}$ \\
\hline 1524.9 & 0.51753 & 0.49844 & 0.47935 & 0.47701 & 0.45939 & 0.49888 \\
\hline 1525.02 & 0.50941 & 0.49544 & 0.48147 & 0.47317 & 0.46254 & 0.49268 \\
\hline 1525.14 & 0.50486 & 0.49111 & 0.47736 & 0.47044 & 0.45853 & 0.49062 \\
\hline 1525.26 & 0.51375 & 0.49504 & 0.47633 & 0.47243 & 0.46196 & 0.49004 \\
\hline 1525.38 & 0.51785 & 0.49733 & 0.47681 & 0.47322 & 0.46254 & 0.49151 \\
\hline 1525.5 & 0.52234 & 0.50184 & 0.48134 & 0.47885 & 0.4703 & 0.49534 \\
\hline 1525.619 & 0.52747 & 0.50358 & 0.47969 & 0.47626 & 0.46483 & 0.49888 \\
\hline 1525.739 & 0.52365 & 0.50136 & 0.47907 & 0.47243 & 0.46512 & 0.49652 \\
\hline 1525.859 & 0.51843 & 0.50033 & 0.48223 & 0.47731 & 0.4654 & 0.49681 \\
\hline 1525.979 & 0.52216 & 0.50271 & 0.48326 & 0.47507 & 0.46655 & 0.49859 \\
\hline 1526.099 & 0.51376 & 0.5012 & 0.48864 & 0.48326 & 0.46943 & 0.49947 \\
\hline 1526.219 & 0.5233 & 0.50652 & 0.48974 & 0.4814 & 0.4703 & 0.50036 \\
\hline & & & & & &
\end{tabular}


Table A2. Cont.

\begin{tabular}{|c|c|c|c|c|c|c|}
\hline Wavelength & NDII & NDII & NDII & NDII & NDII & NDII \\
\hline $\mathrm{nm}$ & $\mathrm{mW}$ & $\mathrm{mW}$ & $\mathrm{mW}$ & $\mathrm{mW}$ & $\mathrm{mW}$ & $\mathrm{mW}$ \\
\hline & DI Water & $25.2 \mathrm{ppm}$ & Water 2 & $43.5 \mathrm{ppm}$ & Water 3 & $64.0 \mathrm{ppm}$ \\
\hline 1526.339 & 0.52529 & 0.50493 & 0.48457 & 0.4814 & 0.46799 & 0.49918 \\
\hline 1526.458 & 0.52852 & 0.50986 & 0.4912 & 0.48256 & 0.47001 & 0.50273 \\
\hline 1526.578 & 0.52389 & 0.50668 & 0.48947 & 0.47955 & 0.46828 & 0.50066 \\
\hline 1526.698 & 0.5253 & 0.50946 & 0.49362 & 0.48682 & 0.46943 & 0.50333 \\
\hline 1526.818 & 0.52945 & 0.51289 & 0.49633 & 0.48466 & 0.47347 & 0.50749 \\
\hline 1526.938 & 0.52949 & 0.51225 & 0.49501 & 0.48581 & 0.47376 & 0.506 \\
\hline 1527.058 & 0.53234 & 0.51329 & 0.49424 & 0.48667 & 0.4755 & 0.50541 \\
\hline 1527.178 & 0.53325 & 0.51465 & 0.49605 & 0.48657 & 0.4755 & 0.506 \\
\hline 1527.298 & 0.53873 & 0.51937 & 0.50001 & 0.49241 & 0.47898 & 0.51137 \\
\hline 1527.418 & 0.54171 & 0.52194 & 0.50217 & 0.49306 & 0.48218 & 0.51316 \\
\hline 1527.537 & 0.53698 & 0.51937 & 0.50176 & 0.49316 & 0.48276 & 0.51047 \\
\hline 1527.657 & 0.54221 & 0.52226 & 0.50231 & 0.49463 & 0.48247 & 0.51257 \\
\hline 1527.777 & 0.5467 & 0.52468 & 0.50266 & 0.49412 & 0.48218 & 0.51346 \\
\hline 1527.897 & 0.54672 & 0.52637 & 0.50602 & 0.49838 & 0.48334 & 0.51436 \\
\hline 1528.017 & 0.54467 & 0.52685 & 0.50903 & 0.49883 & 0.48684 & 0.51766 \\
\hline 1528.137 & 0.54123 & 0.52387 & 0.50651 & 0.49767 & 0.48363 & 0.51406 \\
\hline 1528.257 & 0.5441 & 0.52492 & 0.50574 & 0.49726 & 0.48188 & 0.51496 \\
\hline 1528.377 & 0.54442 & 0.52669 & 0.50896 & 0.49741 & 0.48392 & 0.51466 \\
\hline 1528.496 & 0.54698 & 0.53057 & 0.51416 & 0.50437 & 0.48684 & 0.51856 \\
\hline 1528.616 & 0.54976 & 0.53178 & 0.5138 & 0.50335 & 0.48889 & 0.52006 \\
\hline 1528.736 & 0.55104 & 0.53137 & 0.5117 & 0.5031 & 0.48684 & 0.51856 \\
\hline 1528.856 & 0.54944 & 0.53113 & 0.51282 & 0.50452 & 0.48772 & 0.52036 \\
\hline 1528.976 & 0.55047 & 0.53235 & 0.51423 & 0.503 & 0.48801 & 0.52006 \\
\hline 1529.216 & 0.55852 & 0.53884 & 0.51916 & 0.50962 & 0.49475 & 0.527 \\
\hline 1529.336 & 0.555 & 0.53648 & 0.51796 & 0.50804 & 0.49182 & 0.52338 \\
\hline 1529.456 & 0.5608 & 0.5403 & 0.5198 & 0.51024 & 0.49387 & 0.52579 \\
\hline 1529.575 & 0.5663 & 0.54471 & 0.52312 & 0.51234 & 0.49681 & 0.52942 \\
\hline 1529.695 & 0.56668 & 0.54405 & 0.52142 & 0.51275 & 0.49505 & 0.5273 \\
\hline 1529.815 & 0.56786 & 0.5456 & 0.52334 & 0.51357 & 0.49888 & 0.53003 \\
\hline 1529.935 & 0.56246 & 0.54226 & 0.52206 & 0.51285 & 0.49593 & 0.52821 \\
\hline 1530.055 & 0.55992 & 0.54177 & 0.52362 & 0.51111 & 0.49593 & 0.5264 \\
\hline 1530.175 & 0.56607 & 0.54683 & 0.52759 & 0.51613 & 0.50094 & 0.52851 \\
\hline 1530.295 & 0.56429 & 0.5474 & 0.53051 & 0.51886 & 0.50153 & 0.53063 \\
\hline 1530.415 & 0.56896 & 0.5497 & 0.53044 & 0.51819 & 0.5036 & 0.53154 \\
\hline 1530.534 & 0.56563 & 0.54757 & 0.52951 & 0.51875 & 0.50094 & 0.53033 \\
\hline
\end{tabular}


Table A2. Cont.

\begin{tabular}{|c|c|c|c|c|c|c|}
\hline Wavelength & NDII & NDII & NDII & NDII & NDII & NDII \\
\hline $\mathrm{nm}$ & $\mathrm{mW}$ & $\mathrm{mW}$ & $\mathrm{mW}$ & $\mathrm{mW}$ & $\mathrm{mW}$ & $\mathrm{mW}$ \\
\hline & DI Water & $25.2 \mathrm{ppm}$ & Water 2 & $43.5 \mathrm{ppm}$ & Water 3 & $64.0 \mathrm{ppm}$ \\
\hline 1529.096 & 0.55331 & 0.53405 & 0.51479 & 0.50575 & 0.49123 & 0.52277 \\
\hline 1530.654 & 0.56891 & 0.54978 & 0.53065 & 0.51839 & 0.50272 & 0.53185 \\
\hline 1530.774 & 0.5686 & 0.55084 & 0.53308 & 0.52056 & 0.5042 & 0.53215 \\
\hline 1530.894 & 0.57401 & 0.55429 & 0.53457 & 0.52432 & 0.50538 & 0.53549 \\
\hline 1531.014 & 0.57661 & 0.5547 & 0.53279 & 0.52329 & 0.50509 & 0.53549 \\
\hline 1531.134 & 0.57067 & 0.55273 & 0.53479 & 0.52247 & 0.50568 & 0.53428 \\
\hline 1531.254 & 0.57597 & 0.5552 & 0.53443 & 0.52221 & 0.50686 & 0.5361 \\
\hline 1531.374 & 0.58032 & 0.55866 & 0.537 & 0.52443 & 0.50746 & 0.5361 \\
\hline 1531.494 & 0.57778 & 0.5575 & 0.53722 & 0.52593 & 0.50746 & 0.53702 \\
\hline 1531.613 & 0.58055 & 0.56064 & 0.54073 & 0.52784 & 0.51073 & 0.53885 \\
\hline 1531.733 & 0.57708 & 0.55808 & 0.53908 & 0.52582 & 0.50835 & 0.53732 \\
\hline 1531.853 & 0.58131 & 0.56113 & 0.54095 & 0.5265 & 0.51043 & 0.53732 \\
\hline 1531.973 & 0.57906 & 0.56072 & 0.54238 & 0.52909 & 0.51132 & 0.53915 \\
\hline 1532.093 & 0.58306 & 0.56477 & 0.54648 & 0.53356 & 0.51431 & 0.54129 \\
\hline 1532.213 & 0.58573 & 0.56809 & 0.55045 & 0.53616 & 0.51849 & 0.54435 \\
\hline 1532.333 & 0.58018 & 0.56477 & 0.54936 & 0.53512 & 0.51699 & 0.54129 \\
\hline 1532.453 & 0.58271 & 0.56535 & 0.54799 & 0.53397 & 0.5164 & 0.54098 \\
\hline 1532.572 & 0.58962 & 0.57 & 0.55038 & 0.53679 & 0.51969 & 0.54496 \\
\hline 1532.692 & 0.5882 & 0.57175 & 0.5553 & 0.54196 & 0.52239 & 0.54619 \\
\hline 1532.812 & 0.59301 & 0.57575 & 0.55849 & 0.54468 & 0.5257 & 0.55141 \\
\hline 1532.932 & 0.58986 & 0.572 & 0.55414 & 0.54076 & 0.52089 & 0.5465 \\
\hline 1533.052 & 0.5902 & 0.57141 & 0.55262 & 0.53929 & 0.52239 & 0.54588 \\
\hline 1533.172 & 0.59337 & 0.57575 & 0.55813 & 0.54311 & 0.5257 & 0.5468 \\
\hline 1533.292 & 0.59498 & 0.57608 & 0.55718 & 0.54474 & 0.52449 & 0.5468 \\
\hline 1533.412 & 0.59503 & 0.57658 & 0.55813 & 0.54364 & 0.5263 & 0.54926 \\
\hline 1533.531 & 0.5945 & 0.57566 & 0.55682 & 0.54238 & 0.52359 & 0.54742 \\
\hline 1533.651 & 0.59242 & 0.57433 & 0.55624 & 0.54269 & 0.52299 & 0.54496 \\
\hline 1533.771 & 0.59648 & 0.57901 & 0.56154 & 0.54736 & 0.52901 & 0.54711 \\
\hline 1533.891 & 0.59699 & 0.57934 & 0.56169 & 0.5482 & 0.52871 & 0.54803 \\
\hline 1534.011 & 0.59912 & 0.58303 & 0.56694 & 0.55142 & 0.53204 & 0.55111 \\
\hline 1534.131 & 0.60068 & 0.58286 & 0.56504 & 0.55179 & 0.53204 & 0.54895 \\
\hline 1534.251 & 0.59938 & 0.58261 & 0.56584 & 0.553 & 0.53355 & 0.5508 \\
\hline 1534.371 & 0.60338 & 0.58622 & 0.56906 & 0.55628 & 0.53598 & 0.55234 \\
\hline 1534.49 & 0.604 & 0.58697 & 0.56994 & 0.55553 & 0.53598 & 0.55234 \\
\hline 1534.61 & 0.60676 & 0.58967 & 0.57258 & 0.55808 & 0.53962 & 0.5545 \\
\hline 1534.73 & 0.60637 & 0.5879 & 0.56943 & 0.55866 & 0.53689 & 0.55326 \\
\hline 1534.85 & 0.60773 & 0.58748 & 0.56723 & 0.55601 & 0.53719 & 0.55203 \\
\hline
\end{tabular}


Table A2. Cont.

\begin{tabular}{|c|c|c|c|c|c|c|}
\hline Wavelength & NDII & NDII & NDII & NDII & NDII & NDII \\
\hline $\mathrm{nm}$ & $\mathrm{mW}$ & $\mathrm{mW}$ & $\mathrm{mW}$ & $\mathrm{mW}$ & $\mathrm{mW}$ & $\mathrm{mW}$ \\
\hline & DI Water & $25.2 \mathrm{ppm}$ & Water 2 & $43.5 \mathrm{ppm}$ & Water 3 & $64.0 \mathrm{ppm}$ \\
\hline 1534.97 & 0.60591 & 0.58807 & 0.57023 & 0.55749 & 0.53719 & 0.55234 \\
\hline 1535.09 & 0.60928 & 0.59034 & 0.5714 & 0.55739 & 0.53841 & 0.55265 \\
\hline 1535.21 & 0.61246 & 0.59296 & 0.57346 & 0.55998 & 0.54145 & 0.55419 \\
\hline 1535.33 & 0.61354 & 0.59119 & 0.56884 & 0.55845 & 0.53719 & 0.55111 \\
\hline 1535.45 & 0.61025 & 0.58874 & 0.56723 & 0.55575 & 0.53689 & 0.54772 \\
\hline 1535.569 & 0.61255 & 0.59161 & 0.57067 & 0.55792 & 0.53932 & 0.54895 \\
\hline 1535.689 & 0.61792 & 0.59668 & 0.57544 & 0.56376 & 0.54328 & 0.55357 \\
\hline 1535.809 & 0.61768 & 0.59829 & 0.5789 & 0.56647 & 0.54756 & 0.5579 \\
\hline 1535.929 & 0.61427 & 0.59541 & 0.57655 & 0.56429 & 0.5442 & 0.55296 \\
\hline 1536.049 & 0.61592 & 0.59693 & 0.57794 & 0.56386 & 0.54603 & 0.55357 \\
\hline 1536.169 & 0.62022 & 0.60015 & 0.58008 & 0.5693 & 0.54786 & 0.55419 \\
\hline 1536.289 & 0.61962 & 0.60177 & 0.58392 & 0.57256 & 0.55247 & 0.5579 \\
\hline 1536.409 & 0.61945 & 0.60202 & 0.58459 & 0.57267 & 0.55339 & 0.55914 \\
\hline 1536.528 & 0.61952 & 0.59888 & 0.57824 & 0.56903 & 0.54878 & 0.55512 \\
\hline 1536.648 & 0.61907 & 0.60024 & 0.58141 & 0.57058 & 0.5497 & 0.55512 \\
\hline 1536.768 & 0.6239 & 0.60321 & 0.58252 & 0.57192 & 0.55124 & 0.55481 \\
\hline 1536.888 & 0.62285 & 0.60372 & 0.58459 & 0.5708 & 0.55124 & 0.55419 \\
\hline 1537.008 & 0.62361 & 0.60432 & 0.58503 & 0.57176 & 0.5537 & 0.55728 \\
\hline 1537.128 & 0.62407 & 0.60355 & 0.58303 & 0.5701 & 0.55154 & 0.55419 \\
\hline 1537.248 & 0.62814 & 0.60662 & 0.5851 & 0.57085 & 0.55216 & 0.55419 \\
\hline 1537.368 & 0.62787 & 0.60704 & 0.58621 & 0.5754 & 0.55308 & 0.55542 \\
\hline 1537.488 & 0.63204 & 0.60935 & 0.58666 & 0.57503 & 0.55647 & 0.55573 \\
\hline 1537.607 & 0.63287 & 0.61106 & 0.58925 & 0.5804 & 0.55924 & 0.55883 \\
\hline 1537.727 & 0.63163 & 0.61063 & 0.58963 & 0.58013 & 0.55832 & 0.55666 \\
\hline 1537.847 & 0.63184 & 0.61088 & 0.58992 & 0.58078 & 0.55893 & 0.55635 \\
\hline 1537.967 & 0.63351 & 0.61242 & 0.59133 & 0.58169 & 0.55955 & 0.5579 \\
\hline 1538.087 & 0.63312 & 0.61491 & 0.5967 & 0.58282 & 0.56264 & 0.55914 \\
\hline 1538.207 & 0.63598 & 0.61757 & 0.59916 & 0.58817 & 0.56481 & 0.56038 \\
\hline 1538.327 & 0.63336 & 0.61697 & 0.60058 & 0.58477 & 0.56295 & 0.56255 \\
\hline 1538.447 & 0.63753 & 0.61868 & 0.59983 & 0.58747 & 0.56481 & 0.56255 \\
\hline 1538.566 & 0.63928 & 0.61765 & 0.59602 & 0.58758 & 0.56512 & 0.55914 \\
\hline 1538.686 & 0.64145 & 0.62083 & 0.60021 & 0.59072 & 0.56792 & 0.56038 \\
\hline 1538.806 & 0.64252 & 0.62402 & 0.60552 & 0.59305 & 0.56947 & 0.56348 \\
\hline 1538.926 & 0.63883 & 0.61963 & 0.60043 & 0.58823 & 0.56699 & 0.56069 \\
\hline 1539.046 & 0.64447 & 0.62144 & 0.59841 & 0.58925 & 0.56761 & 0.55976 \\
\hline 1539.166 & 0.63852 & 0.62015 & 0.60178 & 0.58925 & 0.56885 & 0.55945 \\
\hline 1539.286 & 0.64516 & 0.62583 & 0.6065 & 0.59273 & 0.5729 & 0.561 \\
\hline
\end{tabular}


Table A2. Cont.

\begin{tabular}{|c|c|c|c|c|c|c|}
\hline Wavelength & NDII & NDII & NDII & NDII & NDII & NDII \\
\hline $\mathrm{nm}$ & $\mathrm{mW}$ & $\mathrm{mW}$ & $\mathrm{mW}$ & $\mathrm{mW}$ & $\mathrm{mW}$ & $\mathrm{mW}$ \\
\hline & DI Water & $25.2 \mathrm{ppm}$ & Water 2 & $43.5 \mathrm{ppm}$ & Water 3 & $64.0 \mathrm{ppm}$ \\
\hline 1539.406 & 0.6517 & 0.62929 & 0.60688 & 0.59735 & 0.5729 & 0.56193 \\
\hline 1539.526 & 0.65148 & 0.62903 & 0.60658 & 0.59506 & 0.57509 & 0.56131 \\
\hline 1539.645 & 0.64663 & 0.62773 & 0.60883 & 0.59588 & 0.57352 & 0.55976 \\
\hline 1539.765 & 0.64923 & 0.62903 & 0.60883 & 0.59833 & 0.57477 & 0.56224 \\
\hline 1539.885 & 0.65419 & 0.63328 & 0.61237 & 0.60139 & 0.57696 & 0.56441 \\
\hline 1540.005 & 0.65544 & 0.63553 & 0.61562 & 0.60434 & 0.57947 & 0.56784 \\
\hline 1540.125 & 0.65478 & 0.63414 & 0.6135 & 0.60084 & 0.5779 & 0.56659 \\
\hline 1540.245 & 0.65234 & 0.63198 & 0.61162 & 0.60193 & 0.57446 & 0.56379 \\
\hline 1540.365 & 0.65543 & 0.63371 & 0.61199 & 0.60139 & 0.57821 & 0.56317 \\
\hline 1540.484 & 0.65678 & 0.63771 & 0.61864 & 0.60434 & 0.57978 & 0.56628 \\
\hline 1540.604 & 0.65643 & 0.6364 & 0.61637 & 0.60544 & 0.58261 & 0.56846 \\
\hline 1540.724 & 0.66255 & 0.63814 & 0.61373 & 0.60352 & 0.57978 & 0.56472 \\
\hline 1540.844 & 0.65757 & 0.63701 & 0.61645 & 0.60478 & 0.58072 & 0.56628 \\
\hline 1540.964 & 0.6608 & 0.63919 & 0.61758 & 0.60533 & 0.58418 & 0.56753 \\
\hline 1541.084 & 0.66163 & 0.64093 & 0.62023 & 0.60642 & 0.58607 & 0.56784 \\
\hline 1541.204 & 0.66687 & 0.64416 & 0.62145 & 0.60878 & 0.58985 & 0.56784 \\
\hline 1541.324 & 0.66888 & 0.64714 & 0.6254 & 0.61049 & 0.59017 & 0.57033 \\
\hline 1541.444 & 0.6667 & 0.64635 & 0.626 & 0.61401 & 0.59207 & 0.5719 \\
\hline 1541.563 & 0.66733 & 0.6453 & 0.62327 & 0.61137 & 0.58922 & 0.57127 \\
\hline 1541.683 & 0.6656 & 0.64504 & 0.62448 & 0.61208 & 0.59143 & 0.5719 \\
\hline 1541.803 & 0.67077 & 0.64968 & 0.62859 & 0.61467 & 0.59397 & 0.57252 \\
\hline 1541.923 & 0.67036 & 0.64845 & 0.62654 & 0.61462 & 0.59428 & 0.57471 \\
\hline 1542.043 & 0.67384 & 0.65152 & 0.6292 & 0.6171 & 0.59492 & 0.57534 \\
\hline 1542.163 & 0.6695 & 0.64924 & 0.62898 & 0.6155 & 0.59555 & 0.57503 \\
\hline 1542.283 & 0.66931 & 0.65082 & 0.63233 & 0.61809 & 0.59428 & 0.57377 \\
\hline 1542.403 & 0.67271 & 0.65275 & 0.63279 & 0.61688 & 0.59618 & 0.57534 \\
\hline 1542.522 & 0.68003 & 0.65645 & 0.63287 & 0.62009 & 0.59936 & 0.57691 \\
\hline 1542.642 & 0.68196 & 0.65768 & 0.6334 & 0.62347 & 0.60159 & 0.57754 \\
\hline 1542.762 & 0.67908 & 0.65609 & 0.6331 & 0.61809 & 0.60127 & 0.57817 \\
\hline 1542.882 & 0.67796 & 0.65733 & 0.6367 & 0.62286 & 0.60478 & 0.57879 \\
\hline 1543.002 & 0.68338 & 0.66165 & 0.63992 & 0.62485 & 0.6051 & 0.58037 \\
\hline 1543.122 & 0.6828 & 0.66263 & 0.64246 & 0.62674 & 0.60702 & 0.58194 \\
\hline 1543.242 & 0.69194 & 0.66785 & 0.64376 & 0.6307 & 0.6099 & 0.58383 \\
\hline 1543.362 & 0.68645 & 0.66484 & 0.64323 & 0.62814 & 0.60894 & 0.58509 \\
\hline 1543.482 & 0.68584 & 0.66519 & 0.64454 & 0.63181 & 0.6099 & 0.58636 \\
\hline 1543.601 & 0.69129 & 0.6683 & 0.64531 & 0.63209 & 0.61214 & 0.58794 \\
\hline 1543.721 & 0.69079 & 0.66847 & 0.64615 & 0.63394 & 0.61086 & 0.58762 \\
\hline
\end{tabular}


Table A2. Cont.

\begin{tabular}{|c|c|c|c|c|c|c|}
\hline Wavelength & NDII & NDII & NDII & NDII & NDII & NDII \\
\hline $\mathrm{nm}$ & $\mathrm{mW}$ & $\mathrm{mW}$ & $\mathrm{mW}$ & $\mathrm{mW}$ & $\mathrm{mW}$ & $\mathrm{mW}$ \\
\hline & DI Water & $25.2 \mathrm{ppm}$ & Water 2 & $43.5 \mathrm{ppm}$ & Water 3 & $64.0 \mathrm{ppm}$ \\
\hline 1543.841 & 0.6961 & 0.67248 & 0.64886 & 0.63617 & 0.61632 & 0.59238 \\
\hline 1543.961 & 0.69443 & 0.67141 & 0.64839 & 0.63545 & 0.61407 & 0.59016 \\
\hline 1544.081 & 0.6941 & 0.67256 & 0.65102 & 0.6364 & 0.61471 & 0.59269 \\
\hline 1544.201 & 0.69518 & 0.67337 & 0.65156 & 0.63645 & 0.616 & 0.59142 \\
\hline 1544.321 & 0.69867 & 0.67783 & 0.65699 & 0.64178 & 0.6189 & 0.59651 \\
\hline 1544.441 & 0.70701 & 0.68266 & 0.65831 & 0.64296 & 0.62407 & 0.59874 \\
\hline 1544.56 & 0.70144 & 0.67836 & 0.65528 & 0.64257 & 0.62245 & 0.5981 \\
\hline 1544.68 & 0.70117 & 0.67881 & 0.65645 & 0.64313 & 0.62213 & 0.59683 \\
\hline 1544.8 & 0.70591 & 0.6832 & 0.66049 & 0.646 & 0.62569 & 0.5997 \\
\hline 1544.92 & 0.70794 & 0.68562 & 0.6633 & 0.65182 & 0.62991 & 0.60385 \\
\hline 1545.04 & 0.70924 & 0.68732 & 0.6654 & 0.65103 & 0.63024 & 0.60706 \\
\hline 1545.16 & 0.70735 & 0.68661 & 0.66587 & 0.65091 & 0.62991 & 0.60673 \\
\hline 1545.28 & 0.71117 & 0.68993 & 0.66869 & 0.65391 & 0.63056 & 0.6093 \\
\hline 1545.4 & 0.71107 & 0.69074 & 0.67041 & 0.65482 & 0.63284 & 0.61123 \\
\hline 1545.52 & 0.71115 & 0.69192 & 0.67269 & 0.65862 & 0.63317 & 0.61252 \\
\hline 1545.639 & 0.71684 & 0.6948 & 0.67276 & 0.65879 & 0.6348 & 0.61413 \\
\hline 1545.759 & 0.71638 & 0.69426 & 0.67214 & 0.658 & 0.63317 & 0.61252 \\
\hline 1545.879 & 0.71627 & 0.69444 & 0.67261 & 0.65788 & 0.63414 & 0.61316 \\
\hline 1545.999 & 0.71613 & 0.69598 & 0.67583 & 0.65976 & 0.63643 & 0.61478 \\
\hline 1546.119 & 0.71838 & 0.69742 & 0.67646 & 0.66084 & 0.63741 & 0.6151 \\
\hline 1546.239 & 0.72598 & 0.70177 & 0.67756 & 0.66409 & 0.64068 & 0.61898 \\
\hline 1546.359 & 0.72277 & 0.70123 & 0.67969 & 0.66329 & 0.64166 & 0.61962 \\
\hline 1546.479 & 0.7235 & 0.70195 & 0.6804 & 0.66569 & 0.64396 & 0.62092 \\
\hline 1546.598 & 0.725 & 0.70404 & 0.68308 & 0.66723 & 0.64659 & 0.62222 \\
\hline 1546.718 & 0.72222 & 0.70277 & 0.68332 & 0.6686 & 0.64462 & 0.62124 \\
\hline 1546.838 & 0.73196 & 0.7095 & 0.68704 & 0.67038 & 0.64955 & 0.62709 \\
\hline 1546.958 & 0.72775 & 0.70668 & 0.68561 & 0.67043 & 0.64922 & 0.62774 \\
\hline 1547.078 & 0.73353 & 0.71151 & 0.68949 & 0.67313 & 0.65318 & 0.63067 \\
\hline 1547.198 & 0.73033 & 0.71023 & 0.69013 & 0.67336 & 0.65186 & 0.631 \\
\hline 1547.318 & 0.73222 & 0.71169 & 0.69116 & 0.67502 & 0.65252 & 0.63035 \\
\hline 1547.438 & 0.73384 & 0.71489 & 0.69594 & 0.67761 & 0.65649 & 0.63525 \\
\hline 1547.557 & 0.7323 & 0.71352 & 0.69474 & 0.67876 & 0.65285 & 0.63394 \\
\hline 1547.677 & 0.73636 & 0.71599 & 0.69562 & 0.67836 & 0.65517 & 0.63557 \\
\hline 1547.797 & 0.7328 & 0.71397 & 0.69514 & 0.67761 & 0.65616 & 0.63361 \\
\hline 1547.917 & 0.73969 & 0.71901 & 0.69833 & 0.68078 & 0.65948 & 0.63885 \\
\hline 1548.037 & 0.7416 & 0.72112 & 0.70064 & 0.68436 & 0.66147 & 0.64115 \\
\hline 1548.157 & 0.74294 & 0.72287 & 0.7028 & 0.68587 & 0.66413 & 0.64181 \\
\hline
\end{tabular}


Table A2. Cont.

\begin{tabular}{|c|c|c|c|c|c|c|}
\hline Wavelength & NDII & NDII & NDII & NDII & NDII & NDII \\
\hline $\mathrm{nm}$ & $\mathrm{mW}$ & $\mathrm{mW}$ & $\mathrm{mW}$ & $\mathrm{mW}$ & $\mathrm{mW}$ & $\mathrm{mW}$ \\
\hline & DI Water & $25.2 \mathrm{ppm}$ & Water 2 & 43.5 ppm & Water 3 & $64.0 \mathrm{ppm}$ \\
\hline 1548.277 & 0.74673 & 0.72645 & 0.70617 & 0.68824 & 0.66646 & 0.64609 \\
\hline 1548.397 & 0.7439 & 0.72323 & 0.70256 & 0.68592 & 0.66413 & 0.64279 \\
\hline 1548.516 & 0.74703 & 0.72728 & 0.70753 & 0.69033 & 0.66913 & 0.64708 \\
\hline 1548.636 & 0.75317 & 0.73236 & 0.71155 & 0.69469 & 0.67449 & 0.65369 \\
\hline 1548.756 & 0.75597 & 0.73505 & 0.71413 & 0.69795 & 0.67449 & 0.65668 \\
\hline 1548.876 & 0.75013 & 0.73338 & 0.71663 & 0.69755 & 0.67583 & 0.65768 \\
\hline 1548.996 & 0.75196 & 0.73236 & 0.71276 & 0.69906 & 0.67248 & 0.65668 \\
\hline 1549.116 & 0.7561 & 0.73681 & 0.71752 & 0.70064 & 0.67785 & 0.66134 \\
\hline 1549.236 & 0.75338 & 0.73662 & 0.71986 & 0.70251 & 0.67818 & 0.66067 \\
\hline 1549.356 & 0.75768 & 0.73885 & 0.72002 & 0.70316 & 0.67886 & 0.66334 \\
\hline 1549.475 & 0.75861 & 0.74191 & 0.72521 & 0.70767 & 0.68256 & 0.66735 \\
\hline 1549.595 & 0.76076 & 0.74043 & 0.7201 & 0.70538 & 0.67987 & 0.66468 \\
\hline 1549.715 & 0.75874 & 0.74108 & 0.72342 & 0.70673 & 0.68256 & 0.66568 \\
\hline 1549.835 & 0.76532 & 0.74685 & 0.72838 & 0.71249 & 0.68459 & 0.6697 \\
\hline 1549.955 & 0.77077 & 0.75059 & 0.73041 & 0.71426 & 0.69102 & 0.67642 \\
\hline 1550.075 & 0.76692 & 0.74891 & 0.7309 & 0.71503 & 0.69102 & 0.67608 \\
\hline 1550.195 & 0.76662 & 0.74807 & 0.72952 & 0.71467 & 0.69102 & 0.67743 \\
\hline 1550.315 & 0.76931 & 0.75227 & 0.73523 & 0.71733 & 0.69611 & 0.68114 \\
\hline 1550.435 & 0.77103 & 0.75415 & 0.73727 & 0.72206 & 0.69782 & 0.68317 \\
\hline 1550.554 & 0.77644 & 0.75997 & 0.7435 & 0.72521 & 0.70294 & 0.69029 \\
\hline 1550.674 & 0.77855 & 0.76053 & 0.74251 & 0.72604 & 0.70157 & 0.69199 \\
\hline 1550.794 & 0.77572 & 0.75846 & 0.7412 & 0.72467 & 0.70089 & 0.69165 \\
\hline 1550.914 & 0.77438 & 0.75865 & 0.74292 & 0.72723 & 0.70259 & 0.69369 \\
\hline 1551.034 & 0.77538 & 0.761 & 0.74662 & 0.72931 & 0.70328 & 0.69574 \\
\hline 1551.154 & 0.78577 & 0.76751 & 0.74925 & 0.73295 & 0.70979 & 0.70189 \\
\hline 1551.274 & 0.77862 & 0.76373 & 0.74884 & 0.73325 & 0.70704 & 0.69881 \\
\hline 1551.394 & 0.78722 & 0.76647 & 0.74572 & 0.73211 & 0.70807 & 0.70087 \\
\hline 1551.513 & 0.78558 & 0.76779 & 0.75 & 0.73606 & 0.70842 & 0.70327 \\
\hline 1551.633 & 0.78389 & 0.76789 & 0.75189 & 0.73719 & 0.70979 & 0.70395 \\
\hline 1551.753 & 0.79135 & 0.77319 & 0.75503 & 0.73983 & 0.71461 & 0.70911 \\
\hline 1551.873 & 0.79585 & 0.77594 & 0.75603 & 0.74379 & 0.71771 & 0.71117 \\
\hline 1551.993 & 0.79432 & 0.77509 & 0.75586 & 0.74283 & 0.71806 & 0.71428 \\
\hline 1552.113 & 0.7939 & 0.77575 & 0.7576 & 0.74481 & 0.71909 & 0.71394 \\
\hline 1552.233 & 0.7995 & 0.77946 & 0.75942 & 0.74547 & 0.72186 & 0.71878 \\
\hline 1552.353 & 0.80633 & 0.78795 & 0.76957 & 0.75489 & 0.73054 & 0.72573 \\
\hline
\end{tabular}


Table A2. Cont.

\begin{tabular}{|c|c|c|c|c|c|c|}
\hline Wavelength & NDII & NDII & NDII & NDII & NDII & NDII \\
\hline $\mathrm{nm}$ & $\mathrm{mW}$ & $\mathrm{mW}$ & $\mathrm{mW}$ & $\mathrm{mW}$ & $\mathrm{mW}$ & $\mathrm{mW}$ \\
\hline & DI Water & $25.2 \mathrm{ppm}$ & Water 2 & $43.5 \mathrm{ppm}$ & Water 3 & $64.0 \mathrm{ppm}$ \\
\hline 1552.473 & 0.80423 & 0.78661 & 0.76899 & 0.75543 & 0.73228 & 0.72712 \\
\hline 1552.592 & 0.80431 & 0.78853 & 0.77275 & 0.75513 & 0.73124 & 0.72921 \\
\hline 1552.712 & 0.80448 & 0.78757 & 0.77066 & 0.75604 & 0.72915 & 0.73096 \\
\hline 1552.832 & 0.80695 & 0.78968 & 0.77241 & 0.75956 & 0.73368 & 0.73586 \\
\hline 1552.952 & 0.81271 & 0.79591 & 0.77911 & 0.76472 & 0.73752 & 0.73831 \\
\hline 1553.072 & 0.81535 & 0.79639 & 0.77743 & 0.76442 & 0.73787 & 0.74042 \\
\hline 1553.192 & 0.81192 & 0.79514 & 0.77836 & 0.76369 & 0.73647 & 0.73866 \\
\hline 1553.312 & 0.81002 & 0.79553 & 0.78104 & 0.76497 & 0.73822 & 0.73936 \\
\hline 1553.432 & 0.80936 & 0.79659 & 0.78382 & 0.76521 & 0.73998 & 0.74147 \\
\hline 1553.551 & 0.81399 & 0.80063 & 0.78727 & 0.77119 & 0.74348 & 0.74676 \\
\hline 1553.671 & 0.82159 & 0.80536 & 0.78913 & 0.7734 & 0.7477 & 0.75029 \\
\hline 1553.791 & 0.82201 & 0.80671 & 0.79141 & 0.77468 & 0.74947 & 0.75135 \\
\hline 1553.911 & 0.82297 & 0.80584 & 0.78871 & 0.77309 & 0.75088 & 0.75348 \\
\hline 1554.031 & 0.82577 & 0.80952 & 0.79327 & 0.77812 & 0.75477 & 0.75632 \\
\hline 1554.151 & 0.82942 & 0.81457 & 0.79972 & 0.78353 & 0.75866 & 0.76023 \\
\hline 1554.271 & 0.83184 & 0.81612 & 0.8004 & 0.78409 & 0.75902 & 0.76487 \\
\hline 1554.391 & 0.83197 & 0.81661 & 0.80125 & 0.78563 & 0.75866 & 0.76701 \\
\hline 1554.51 & 0.82876 & 0.81428 & 0.7998 & 0.78403 & 0.75866 & 0.76665 \\
\hline 1554.63 & 0.83265 & 0.81895 & 0.80525 & 0.78754 & 0.76186 & 0.76916 \\
\hline 1554.75 & 0.83875 & 0.82217 & 0.80559 & 0.7886 & 0.76328 & 0.76987 \\
\hline 1554.87 & 0.84071 & 0.8252 & 0.80969 & 0.79256 & 0.76471 & 0.7767 \\
\hline 1554.99 & 0.83796 & 0.82344 & 0.80892 & 0.79429 & 0.76649 & 0.77562 \\
\hline 1555.11 & 0.83917 & 0.8249 & 0.81063 & 0.79212 & 0.76684 & 0.77598 \\
\hline 1555.23 & 0.84242 & 0.82794 & 0.81346 & 0.79796 & 0.76863 & 0.77886 \\
\hline 1555.35 & 0.8488 & 0.83216 & 0.81552 & 0.79995 & 0.77184 & 0.78138 \\
\hline 1555.469 & 0.84929 & 0.83481 & 0.82033 & 0.8045 & 0.77579 & 0.78536 \\
\hline 1555.589 & 0.85237 & 0.83609 & 0.81981 & 0.80494 & 0.7783 & 0.78753 \\
\hline 1555.709 & 0.85114 & 0.83668 & 0.82222 & 0.80644 & 0.78046 & 0.78862 \\
\hline 1555.829 & 0.85743 & 0.84112 & 0.82481 & 0.81082 & 0.78334 & 0.79188 \\
\hline 1555.949 & 0.86059 & 0.84369 & 0.82679 & 0.80982 & 0.78514 & 0.79479 \\
\hline 1556.069 & 0.85977 & 0.84497 & 0.83017 & 0.81496 & 0.78658 & 0.79698 \\
\hline 1556.189 & 0.85971 & 0.84507 & 0.83043 & 0.81427 & 0.78948 & 0.7999 \\
\hline 1556.309 & 0.86231 & 0.84784 & 0.83337 & 0.81659 & 0.78984 & 0.80173 \\
\hline 1556.429 & 0.86566 & 0.84943 & 0.8332 & 0.81968 & 0.78984 & 0.80173 \\
\hline 1556.548 & 0.86356 & 0.85003 & 0.8365 & 0.8193 & 0.79092 & 0.80429 \\
\hline 1556.668 & 0.86858 & 0.8538 & 0.83902 & 0.8229 & 0.7931 & 0.80795 \\
\hline 1556.788 & 0.87084 & 0.8551 & 0.83936 & 0.82302 & 0.79673 & 0.80795 \\
\hline
\end{tabular}


Table A2. Cont.

\begin{tabular}{|c|c|c|c|c|c|c|}
\hline Wavelength & NDII & NDII & NDII & NDII & NDII & NDII \\
\hline nm & $\mathbf{m W}$ & $\mathrm{mW}$ & $\mathbf{m W}$ & $\mathrm{mW}$ & $\mathrm{mW}$ & $\mathrm{mW}$ \\
\hline & DI Water & $25.2 \mathrm{ppm}$ & Water 2 & 43.5 ppm & Water 3 & $64.0 \mathrm{ppm}$ \\
\hline 1556.908 & 0.87313 & 0.85838 & 0.84363 & 0.82758 & 0.79964 & 0.81126 \\
\hline 1557.028 & 0.87394 & 0.85918 & 0.84442 & 0.82929 & 0.8 & 0.81163 \\
\hline 1557.148 & 0.87538 & 0.86108 & 0.84678 & 0.82998 & 0.8011 & 0.81568 \\
\hline 1557.268 & 0.88238 & 0.86668 & 0.85098 & 0.83373 & 0.80438 & 0.81863 \\
\hline 1557.388 & 0.88802 & 0.87099 & 0.85396 & 0.83863 & 0.80914 & 0.82159 \\
\hline 1557.507 & 0.89148 & 0.8725 & 0.85352 & 0.8415 & 0.81134 & 0.82345 \\
\hline 1557.627 & 0.88677 & 0.87019 & 0.85361 & 0.83787 & 0.81134 & 0.82345 \\
\hline 1557.747 & 0.89029 & 0.873 & 0.85571 & 0.84182 & 0.81317 & 0.82419 \\
\hline 1557.867 & 0.89629 & 0.87895 & 0.86161 & 0.84412 & 0.81795 & 0.8279 \\
\hline 1557.987 & 0.89623 & 0.87976 & 0.86329 & 0.84489 & 0.82164 & 0.83013 \\
\hline 1558.107 & 0.89502 & 0.88238 & 0.86974 & 0.85156 & 0.82423 & 0.83684 \\
\hline 1558.227 & 0.89439 & 0.88127 & 0.86815 & 0.84969 & 0.82312 & 0.83684 \\
\hline 1558.347 & 0.90021 & 0.88471 & 0.86921 & 0.85233 & 0.8246 & 0.83684 \\
\hline 1558.467 & 0.90288 & 0.88755 & 0.87222 & 0.85246 & 0.82682 & 0.83984 \\
\hline 1558.586 & 0.90357 & 0.88927 & 0.87497 & 0.85877 & 0.82979 & 0.84246 \\
\hline 1558.706 & 0.91208 & 0.89344 & 0.8748 & 0.85677 & 0.8309 & 0.84246 \\
\hline 1558.826 & 0.90884 & 0.89364 & 0.87844 & 0.86245 & 0.83462 & 0.84509 \\
\hline 1558.946 & 0.91572 & 0.89904 & 0.88236 & 0.86484 & 0.83909 & 0.8496 \\
\hline 1559.066 & 0.92115 & 0.9018 & 0.88245 & 0.86504 & 0.83947 & 0.8496 \\
\hline 1559.186 & 0.92187 & 0.90426 & 0.88665 & 0.87029 & 0.84021 & 0.85413 \\
\hline 1559.306 & 0.92322 & 0.90641 & 0.8896 & 0.8725 & 0.8462 & 0.85602 \\
\hline 1559.426 & 0.92339 & 0.90753 & 0.89167 & 0.87699 & 0.8492 & 0.85753 \\
\hline 1559.545 & 0.92797 & 0.91031 & 0.89265 & 0.87915 & 0.85409 & 0.8636 \\
\hline 1559.665 & 0.92835 & 0.91041 & 0.89247 & 0.87908 & 0.85259 & 0.86246 \\
\hline 1559.785 & 0.93072 & 0.9138 & 0.89688 & 0.8815 & 0.85711 & 0.8655 \\
\hline 1559.905 & 0.93318 & 0.9171 & 0.90102 & 0.88333 & 0.85975 & 0.8693 \\
\hline 1560.025 & 0.93376 & 0.91906 & 0.90436 & 0.88602 & 0.85975 & 0.86969 \\
\hline 1560.145 & 0.9364 & 0.92133 & 0.90626 & 0.88818 & 0.86429 & 0.87274 \\
\hline 1560.265 & 0.93608 & 0.9204 & 0.90472 & 0.8868 & 0.8624 & 0.87121 \\
\hline 1560.385 & 0.93648 & 0.92123 & 0.90598 & 0.89022 & 0.86392 & 0.87159 \\
\hline 1560.505 & 0.94176 & 0.9265 & 0.91124 & 0.89213 & 0.86505 & 0.87503 \\
\hline 1560.624 & 0.94274 & 0.92785 & 0.91296 & 0.89555 & 0.86847 & 0.87809 \\
\hline 1560.744 & 0.94275 & 0.92858 & 0.91441 & 0.89773 & 0.87075 & 0.88193 \\
\hline 1560.864 & 0.94308 & 0.92879 & 0.9145 & 0.89991 & 0.87227 & 0.87809 \\
\hline 1560.984 & 0.94631 & 0.93159 & 0.91687 & 0.90308 & 0.87266 & 0.8827 \\
\hline 1561.104 & 0.94526 & 0.93325 & 0.92124 & 0.90302 & 0.87647 & 0.88385 \\
\hline
\end{tabular}


Table A2. Cont.

\begin{tabular}{|c|c|c|c|c|c|c|}
\hline Wavelength & NDII & NDII & NDII & NDII & NDII & NDII \\
\hline $\mathrm{nm}$ & $\mathrm{mW}$ & $\mathrm{mW}$ & $\mathrm{mW}$ & $\mathrm{mW}$ & $\mathrm{mW}$ & $\mathrm{mW}$ \\
\hline & DI Water & $25.2 \mathrm{ppm}$ & Water 2 & $43.5 \mathrm{ppm}$ & Water 3 & $64.0 \mathrm{ppm}$ \\
\hline 1561.224 & 0.95679 & 0.93888 & 0.92097 & 0.90806 & 0.88259 & 0.88847 \\
\hline 1561.344 & 0.95615 & 0.94066 & 0.92517 & 0.90892 & 0.88489 & 0.88963 \\
\hline 1561.464 & 0.95451 & 0.93961 & 0.92471 & 0.90925 & 0.88297 & 0.89002 \\
\hline 1561.583 & 0.95615 & 0.94139 & 0.92663 & 0.91211 & 0.88604 & 0.89388 \\
\hline 1561.703 & 0.95373 & 0.9416 & 0.92947 & 0.91264 & 0.88835 & 0.89272 \\
\hline 1561.823 & 0.95509 & 0.94379 & 0.93249 & 0.91591 & 0.8922 & 0.89775 \\
\hline 1561.943 & 0.95365 & 0.94243 & 0.93121 & 0.91711 & 0.8895 & 0.89775 \\
\hline 1562.063 & 0.95803 & 0.94421 & 0.93039 & 0.91598 & 0.88874 & 0.8962 \\
\hline 1562.183 & 0.95876 & 0.94526 & 0.93176 & 0.91564 & 0.89105 & 0.89737 \\
\hline 1562.303 & 0.96019 & 0.94662 & 0.93305 & 0.92045 & 0.89452 & 0.89853 \\
\hline 1562.423 & 0.96544 & 0.95145 & 0.93746 & 0.92139 & 0.89683 & 0.9028 \\
\hline 1562.542 & 0.96202 & 0.95029 & 0.93856 & 0.92279 & 0.89722 & 0.90164 \\
\hline 1562.662 & 0.96345 & 0.95239 & 0.94133 & 0.92514 & 0.90303 & 0.90436 \\
\hline 1562.782 & 0.96405 & 0.95292 & 0.94179 & 0.92682 & 0.90303 & 0.90475 \\
\hline 1562.902 & 0.96694 & 0.95418 & 0.94142 & 0.92803 & 0.90381 & 0.9067 \\
\hline 1563.022 & 0.97088 & 0.95892 & 0.94696 & 0.93139 & 0.90964 & 0.91177 \\
\hline 1563.142 & 0.97729 & 0.96125 & 0.94521 & 0.93327 & 0.91042 & 0.91099 \\
\hline 1563.262 & 0.98311 & 0.96601 & 0.94891 & 0.93698 & 0.91588 & 0.91412 \\
\hline 1563.382 & 0.97743 & 0.96326 & 0.94909 & 0.93638 & 0.91471 & 0.91334 \\
\hline 1563.501 & 0.98363 & 0.96738 & 0.95113 & 0.93881 & 0.9198 & 0.91568 \\
\hline 1563.621 & 0.97304 & 0.96283 & 0.95262 & 0.93969 & 0.91549 & 0.91647 \\
\hline 1563.741 & 0.97548 & 0.96558 & 0.95568 & 0.94097 & 0.91862 & 0.91725 \\
\hline 1563.861 & 0.97912 & 0.96791 & 0.9567 & 0.93982 & 0.92176 & 0.91961 \\
\hline 1563.981 & 0.97801 & 0.96601 & 0.95401 & 0.93901 & 0.9194 & 0.91647 \\
\hline 1564.101 & 0.98781 & 0.97407 & 0.96033 & 0.94436 & 0.92529 & 0.92 \\
\hline 1564.221 & 0.98063 & 0.97067 & 0.96071 & 0.94619 & 0.92529 & 0.92157 \\
\hline 1564.341 & 0.97838 & 0.97057 & 0.96276 & 0.94762 & 0.92765 & 0.92393 \\
\hline 1564.461 & 0.98368 & 0.97322 & 0.96276 & 0.94898 & 0.92883 & 0.92354 \\
\hline 1564.58 & 0.9874 & 0.97695 & 0.9665 & 0.9534 & 0.93278 & 0.92472 \\
\hline 1564.7 & 0.99038 & 0.98036 & 0.97034 & 0.9577 & 0.93831 & 0.93104 \\
\hline 1564.82 & 0.9892 & 0.97855 & 0.9679 & 0.95681 & 0.93633 & 0.93064 \\
\hline 1564.94 & 0.98566 & 0.97823 & 0.9708 & 0.95872 & 0.9399 & 0.93183 \\
\hline 1565.06 & 0.98168 & 0.97493 & 0.96818 & 0.95879 & 0.93792 & 0.92867 \\
\hline 1565.18 & 0.9932 & 0.98261 & 0.97202 & 0.96242 & 0.94347 & 0.93301 \\
\hline 1565.3 & 0.99244 & 0.98303 & 0.97362 & 0.9618 & 0.94506 & 0.9346 \\
\hline 1565.42 & 0.98877 & 0.98143 & 0.97409 & 0.96214 & 0.94625 & 0.93618 \\
\hline
\end{tabular}


Table A2. Cont.

\begin{tabular}{|c|c|c|c|c|c|c|}
\hline Wavelength & NDII & NDII & NDII & NDII & NDII & NDII \\
\hline nm & $\mathbf{m W}$ & $\mathbf{m W}$ & $\mathbf{m W}$ & $\mathrm{mW}$ & $\mathrm{mW}$ & $\mathrm{mW}$ \\
\hline & DI Water & $25.2 \mathrm{ppm}$ & Water 2 & 43.5 ppm & Water 3 & $64.0 \mathrm{ppm}$ \\
\hline 1565.539 & 0.99264 & 0.98421 & 0.97578 & 0.96276 & 0.94705 & 0.93658 \\
\hline 1565.659 & 0.99326 & 0.98325 & 0.97324 & 0.96132 & 0.94785 & 0.93698 \\
\hline 1565.779 & 0.99524 & 0.98678 & 0.97832 & 0.96694 & 0.95263 & 0.94175 \\
\hline 1565.899 & 0.99464 & 0.98549 & 0.97634 & 0.96846 & 0.95183 & 0.93896 \\
\hline 1566.019 & 0.99779 & 0.98796 & 0.97813 & 0.96585 & 0.95383 & 0.94254 \\
\hline 1566.139 & 0.9967 & 0.98817 & 0.97964 & 0.97052 & 0.95383 & 0.94214 \\
\hline 1566.259 & 1.001 & 0.99107 & 0.98114 & 0.97382 & 0.95663 & 0.94573 \\
\hline 1566.379 & 0.99199 & 0.98817 & 0.98435 & 0.97465 & 0.95863 & 0.94932 \\
\hline 1566.499 & 0.99769 & 0.99107 & 0.98445 & 0.97486 & 0.96064 & 0.94772 \\
\hline 1566.618 & 0.99921 & 0.99386 & 0.98851 & 0.97893 & 0.96506 & 0.95412 \\
\hline 1566.738 & 1.00015 & 0.99386 & 0.98757 & 0.98135 & 0.96426 & 0.95452 \\
\hline 1566.858 & 0.99771 & 0.99472 & 0.99173 & 0.98218 & 0.96909 & 0.95653 \\
\hline 1566.978 & 0.99288 & 0.99117 & 0.98946 & 0.98412 & 0.96949 & 0.95854 \\
\hline 1567.098 & 0.99197 & 0.99408 & 0.99619 & 0.98641 & 0.97595 & 0.96498 \\
\hline 1567.218 & 0.99499 & 0.99526 & 0.99553 & 0.98982 & 0.97393 & 0.9674 \\
\hline 1567.338 & 0.99414 & 0.99289 & 0.99164 & 0.98336 & 0.97474 & 0.96337 \\
\hline 1567.458 & 0.99635 & 0.99537 & 0.99439 & 0.98697 & 0.97757 & 0.96619 \\
\hline 1567.577 & 0.99623 & 0.99332 & 0.99041 & 0.98551 & 0.97636 & 0.96538 \\
\hline 1567.697 & 0.99897 & 0.99644 & 0.99391 & 0.98836 & 0.97879 & 0.9678 \\
\hline 1567.817 & 0.99721 & 0.99547 & 0.99373 & 0.98829 & 0.97757 & 0.96699 \\
\hline 1567.937 & 0.99596 & 0.99451 & 0.99306 & 0.98683 & 0.97717 & 0.9678 \\
\hline 1568.057 & 0.99614 & 0.99569 & 0.99524 & 0.99058 & 0.9792 & 0.96982 \\
\hline 1568.177 & 0.99668 & 0.99634 & 0.996 & 0.99051 & 0.98326 & 0.97225 \\
\hline 1568.297 & 0.99786 & 0.99817 & 0.99848 & 0.99211 & 0.98285 & 0.97347 \\
\hline 1568.417 & 1.00069 & 0.99892 & 0.99715 & 0.99267 & 0.98285 & 0.97185 \\
\hline 1568.536 & 0.99799 & 0.99795 & 0.99791 & 0.99609 & 0.98814 & 0.97671 \\
\hline 1568.656 & 0.99838 & 0.99881 & 0.99924 & 0.99902 & 0.98896 & 0.97712 \\
\hline 1568.776 & 1.00095 & 1 & 0.99905 & 0.9979 & 0.99018 & 0.97996 \\
\hline 1568.896 & 0.99784 & 0.99892 & 1 & 1 & 0.99222 & 0.98199 \\
\hline 1569.016 & 0.99719 & 0.99774 & 0.99829 & 0.99553 & 0.99304 & 0.97996 \\
\hline 1569.136 & 0.99194 & 0.99483 & 0.99772 & 0.99567 & 0.991 & 0.97793 \\
\hline 1569.256 & 0.99605 & 0.99655 & 0.99705 & 0.99595 & 0.99263 & 0.98199 \\
\hline 1569.376 & 0.9924 & 0.99558 & 0.99876 & 0.99483 & 0.99426 & 0.98281 \\
\hline 1569.495 & 0.98929 & 0.99236 & 0.99543 & 0.99518 & 0.99222 & 0.9824 \\
\hline 1569.615 & 0.9903 & 0.99225 & 0.9942 & 0.99211 & 0.99304 & 0.98281 \\
\hline 1569.735 & 0.99004 & 0.99193 & 0.99382 & 0.99616 & 0.99426 & 0.98403 \\
\hline 1569.855 & 0.9858 & 0.98967 & 0.99354 & 0.99204 & 0.99222 & 0.98159 \\
\hline
\end{tabular}


Table A2. Cont.

\begin{tabular}{|c|c|c|c|c|c|c|}
\hline Wavelength & NDII & NDII & NDII & NDII & NDII & NDII \\
\hline $\mathrm{nm}$ & $\mathrm{mW}$ & $\mathrm{mW}$ & $\mathrm{mW}$ & $\mathrm{mW}$ & $\mathrm{mW}$ & $\mathrm{mW}$ \\
\hline & DI Water & $25.2 \mathrm{ppm}$ & Water 2 & 43.5 ppm & Water 3 & $64.0 \mathrm{ppm}$ \\
\hline 1569.975 & 0.98847 & 0.99214 & 0.99581 & 0.99462 & 0.99549 & 0.98485 \\
\hline 1570.095 & 0.98734 & 0.99096 & 0.99458 & 0.9926 & 0.99508 & 0.98566 \\
\hline 1570.215 & 0.98598 & 0.99085 & 0.99572 & 0.99476 & 0.9959 & 0.98852 \\
\hline 1570.335 & 0.98584 & 0.98817 & 0.9905 & 0.99476 & 0.99304 & 0.98444 \\
\hline 1570.455 & 0.98741 & 0.99085 & 0.99429 & 0.99581 & 0.99754 & 0.98975 \\
\hline 1570.574 & 0.98662 & 0.98989 & 0.99316 & 0.99609 & 0.99631 & 0.9877 \\
\hline 1570.694 & 0.98788 & 0.99085 & 0.99382 & 0.9972 & 0.99713 & 0.99097 \\
\hline 1570.814 & 0.98669 & 0.98978 & 0.99287 & 0.9986 & 0.99795 & 0.99302 \\
\hline 1570.934 & 0.99066 & 0.99096 & 0.99126 & 0.99609 & 0.99672 & 0.99261 \\
\hline 1571.054 & 0.98401 & 0.98806 & 0.99211 & 0.99797 & 0.99959 & 0.99466 \\
\hline 1571.174 & 0.98135 & 0.98635 & 0.99135 & 0.9963 & 1 & 0.99548 \\
\hline 1571.294 & 0.98314 & 0.98796 & 0.99278 & 0.99316 & 0.99959 & 0.99589 \\
\hline 1571.414 & 0.98806 & 0.98914 & 0.99022 & 0.99448 & 0.99918 & 0.99753 \\
\hline 1571.533 & 0.97941 & 0.98207 & 0.98473 & 0.98933 & 0.99713 & 0.99261 \\
\hline 1571.653 & 0.97832 & 0.98228 & 0.98624 & 0.99128 & 0.99959 & 0.99425 \\
\hline 1571.773 & 0.97741 & 0.98154 & 0.98567 & 0.99177 & 0.99959 & 0.99507 \\
\hline 1571.893 & 0.97334 & 0.97908 & 0.98482 & 0.99093 & 0.99836 & 0.99753 \\
\hline 1572.013 & 0.97069 & 0.97738 & 0.98407 & 0.98996 & 0.99754 & 0.99548 \\
\hline 1572.133 & 0.97226 & 0.97642 & 0.98058 & 0.98745 & 0.99713 & 0.99384 \\
\hline 1572.253 & 0.97158 & 0.97674 & 0.9819 & 0.98933 & 0.99836 & 0.99794 \\
\hline 1572.373 & 0.97191 & 0.97695 & 0.98199 & 0.99024 & 0.99795 & 0.99753 \\
\hline 1572.493 & 0.97258 & 0.97738 & 0.98218 & 0.99204 & 0.99918 & 0.99959 \\
\hline 1572.612 & 0.96465 & 0.97078 & 0.97691 & 0.98523 & 0.99631 & 0.99836 \\
\hline 1572.732 & 0.96542 & 0.97248 & 0.97954 & 0.9869 & 0.99795 & 1 \\
\hline 1572.852 & 0.96262 & 0.96887 & 0.97512 & 0.98752 & 0.99754 & 0.99877 \\
\hline 1572.972 & 0.96064 & 0.9676 & 0.97456 & 0.98489 & 0.99631 & 0.99794 \\
\hline 1573.092 & 0.95602 & 0.96463 & 0.97324 & 0.98475 & 0.99426 & 0.99877 \\
\hline 1573.212 & 0.95367 & 0.96177 & 0.96987 & 0.98108 & 0.99059 & 0.99794 \\
\hline 1573.332 & 0.95202 & 0.95935 & 0.96668 & 0.97783 & 0.99018 & 0.9963 \\
\hline 1573.452 & 0.9468 & 0.9566 & 0.9664 & 0.97479 & 0.98896 & 0.99589 \\
\hline 1573.571 & 0.94577 & 0.95534 & 0.96491 & 0.97665 & 0.991 & 0.9963 \\
\hline 1573.691 & 0.95032 & 0.95766 & 0.965 & 0.9772 & 0.99345 & 0.9963 \\
\hline 1573.811 & 0.95038 & 0.95755 & 0.96472 & 0.97624 & 0.99222 & 0.99753 \\
\hline 1573.931 & 0.94029 & 0.95092 & 0.96155 & 0.973 & 0.98733 & 0.99548 \\
\hline 1574.051 & 0.93684 & 0.9484 & 0.95996 & 0.97017 & 0.98814 & 0.99179 \\
\hline 1574.171 & 0.94019 & 0.9504 & 0.96061 & 0.97403 & 0.98936 & 0.99589 \\
\hline 1574.291 & 0.93787 & 0.94966 & 0.96145 & 0.97176 & 0.98896 & 0.99548 \\
\hline
\end{tabular}


Table A2. Cont.

\begin{tabular}{|c|c|c|c|c|c|c|}
\hline Wavelength & NDII & NDII & NDII & NDII & NDII & NDII \\
\hline $\mathrm{nm}$ & $\mathrm{mW}$ & $\mathrm{mW}$ & $\mathrm{mW}$ & $\mathrm{mW}$ & $\mathrm{mW}$ & $\mathrm{mW}$ \\
\hline & DI Water & $25.2 \mathrm{ppm}$ & Water 2 & $43.5 \mathrm{ppm}$ & Water 3 & $64.0 \mathrm{ppm}$ \\
\hline 1574.411 & 0.94057 & 0.9505 & 0.96043 & 0.975 & 0.99018 & 1 \\
\hline 1574.531 & 0.93297 & 0.94442 & 0.95587 & 0.96969 & 0.98855 & 0.99794 \\
\hline 1574.65 & 0.93265 & 0.94212 & 0.95159 & 0.96639 & 0.98285 & 0.99179 \\
\hline 1574.77 & 0.93295 & 0.94306 & 0.95317 & 0.96887 & 0.9861 & 0.99753 \\
\hline 1574.89 & 0.92962 & 0.94107 & 0.95252 & 0.96777 & 0.9861 & 0.99712 \\
\hline 1575.01 & 0.9244 & 0.93846 & 0.95252 & 0.96708 & 0.98488 & 0.9963 \\
\hline 1575.13 & 0.92508 & 0.93607 & 0.94706 & 0.96112 & 0.98326 & 0.99425 \\
\hline 1575.25 & 0.91493 & 0.9291 & 0.94327 & 0.95544 & 0.97555 & 0.98689 \\
\hline 1575.37 & 0.91653 & 0.92879 & 0.94105 & 0.95565 & 0.97757 & 0.98689 \\
\hline 1575.49 & 0.90974 & 0.92443 & 0.93912 & 0.95272 & 0.97515 & 0.98444 \\
\hline 1575.609 & 0.9104 & 0.92278 & 0.93516 & 0.95142 & 0.97717 & 0.98648 \\
\hline 1575.729 & 0.90584 & 0.91926 & 0.93268 & 0.9466 & 0.9707 & 0.98118 \\
\hline 1575.849 & 0.90625 & 0.91864 & 0.93103 & 0.947 & 0.96828 & 0.98281 \\
\hline 1575.969 & 0.89911 & 0.9136 & 0.92809 & 0.94355 & 0.96909 & 0.98077 \\
\hline 1576.089 & 0.89348 & 0.90969 & 0.9259 & 0.94409 & 0.96868 & 0.97833 \\
\hline 1576.209 & 0.89142 & 0.90866 & 0.9259 & 0.93867 & 0.96868 & 0.97955 \\
\hline 1576.329 & 0.89593 & 0.90877 & 0.92161 & 0.94131 & 0.96586 & 0.97833 \\
\hline 1576.449 & 0.89189 & 0.90661 & 0.92133 & 0.93766 & 0.96225 & 0.97996 \\
\hline 1576.568 & 0.88046 & 0.89853 & 0.9166 & 0.93152 & 0.95743 & 0.97428 \\
\hline 1576.688 & 0.87887 & 0.89619 & 0.91351 & 0.93307 & 0.95783 & 0.97387 \\
\hline 1576.808 & 0.878 & 0.89344 & 0.90888 & 0.92581 & 0.95343 & 0.97144 \\
\hline 1576.928 & 0.87722 & 0.89323 & 0.90924 & 0.9293 & 0.95423 & 0.97104 \\
\hline 1577.048 & 0.87559 & 0.89029 & 0.90499 & 0.92554 & 0.95303 & 0.97144 \\
\hline 1577.168 & 0.86178 & 0.87996 & 0.89814 & 0.91965 & 0.94625 & 0.9674 \\
\hline 1577.288 & 0.86426 & 0.87895 & 0.89364 & 0.91431 & 0.94427 & 0.96337 \\
\hline 1577.408 & 0.85509 & 0.87522 & 0.89535 & 0.91145 & 0.94347 & 0.96055 \\
\hline 1577.527 & 0.85992 & 0.87804 & 0.89616 & 0.91524 & 0.94546 & 0.96296 \\
\hline 1577.647 & 0.84969 & 0.87059 & 0.89149 & 0.91052 & 0.93911 & 0.95814 \\
\hline 1577.767 & 0.84204 & 0.86278 & 0.88352 & 0.9054 & 0.9308 & 0.95292 \\
\hline 1577.887 & 0.8372 & 0.85938 & 0.88156 & 0.90136 & 0.93041 & 0.94892 \\
\hline 1578.007 & 0.83583 & 0.85749 & 0.87915 & 0.89832 & 0.92883 & 0.94733 \\
\hline 1578.127 & 0.83787 & 0.85838 & 0.87889 & 0.89971 & 0.92962 & 0.95052 \\
\hline 1578.247 & 0.83912 & 0.85589 & 0.87266 & 0.89806 & 0.92608 & 0.94613 \\
\hline 1578.367 & 0.83033 & 0.85052 & 0.87071 & 0.89305 & 0.92529 & 0.94493 \\
\hline 1578.487 & 0.82536 & 0.84547 & 0.86558 & 0.88818 & 0.91784 & 0.93936 \\
\hline 1578.606 & 0.82465 & 0.84388 & 0.86311 & 0.88792 & 0.91784 & 0.93976 \\
\hline
\end{tabular}


Table A2. Cont.

\begin{tabular}{|c|c|c|c|c|c|c|}
\hline Wavelength & NDII & NDII & NDII & NDII & NDII & NDII \\
\hline $\mathrm{nm}$ & $\mathrm{mW}$ & $\mathrm{mW}$ & $\mathrm{mW}$ & $\mathrm{mW}$ & $\mathrm{mW}$ & $\mathrm{mW}$ \\
\hline & DI Water & $25.2 \mathrm{ppm}$ & Water 2 & $43.5 \mathrm{ppm}$ & Water 3 & $64.0 \mathrm{ppm}$ \\
\hline 1578.726 & 0.81845 & 0.84003 & 0.86161 & 0.8849 & 0.91432 & 0.93737 \\
\hline 1578.846 & 0.81583 & 0.83806 & 0.86029 & 0.88392 & 0.91549 & 0.93737 \\
\hline 1578.966 & 0.81443 & 0.8356 & 0.85677 & 0.87673 & 0.91198 & 0.93381 \\
\hline 1579.086 & 0.80873 & 0.82784 & 0.84695 & 0.87146 & 0.90575 & 0.92788 \\
\hline 1579.206 & 0.80486 & 0.82451 & 0.84416 & 0.86685 & 0.9007 & 0.92315 \\
\hline 1579.326 & 0.80134 & 0.82266 & 0.84398 & 0.86776 & 0.89915 & 0.92354 \\
\hline 1579.446 & 0.79802 & 0.82109 & 0.84416 & 0.86523 & 0.89877 & 0.92118 \\
\hline 1579.565 & 0.78503 & 0.8102 & 0.83537 & 0.85857 & 0.89374 & 0.91608 \\
\hline 1579.685 & 0.78422 & 0.80633 & 0.82844 & 0.85342 & 0.89027 & 0.91255 \\
\hline 1579.805 & 0.78272 & 0.80536 & 0.828 & 0.85278 & 0.8872 & 0.91099 \\
\hline 1579.925 & 0.77712 & 0.80092 & 0.82472 & 0.84963 & 0.88413 & 0.90592 \\
\hline 1580.045 & 0.77461 & 0.79764 & 0.82067 & 0.84604 & 0.88029 & 0.90202 \\
\hline 1580.165 & 0.77084 & 0.79438 & 0.81792 & 0.84502 & 0.87762 & 0.90241 \\
\hline 1580.285 & 0.76761 & 0.78968 & 0.81175 & 0.83984 & 0.87647 & 0.89931 \\
\hline 1580.405 & 0.75576 & 0.78136 & 0.80696 & 0.83297 & 0.86923 & 0.89272 \\
\hline 1580.525 & 0.75866 & 0.78089 & 0.80312 & 0.82789 & 0.86619 & 0.88847 \\
\hline 1580.644 & 0.75028 & 0.77632 & 0.80236 & 0.82745 & 0.86429 & 0.88847 \\
\hline 1580.764 & 0.74705 & 0.773 & 0.79895 & 0.8246 & 0.8624 & 0.88539 \\
\hline 1580.884 & 0.74328 & 0.76921 & 0.79514 & 0.82113 & 0.86051 & 0.88308 \\
\hline 1581.004 & 0.74383 & 0.76534 & 0.78685 & 0.81615 & 0.8507 & 0.87656 \\
\hline 1581.124 & 0.73345 & 0.75771 & 0.78197 & 0.80994 & 0.8477 & 0.86702 \\
\hline 1581.244 & 0.72756 & 0.75405 & 0.78054 & 0.80737 & 0.84245 & 0.86588 \\
\hline 1581.364 & 0.72969 & 0.75583 & 0.78197 & 0.80712 & 0.84433 & 0.86778 \\
\hline 1581.484 & 0.71835 & 0.74555 & 0.77275 & 0.7987 & 0.83723 & 0.86094 \\
\hline 1581.603 & 0.71562 & 0.74043 & 0.76524 & 0.79467 & 0.83239 & 0.85602 \\
\hline 1581.723 & 0.71143 & 0.73746 & 0.76349 & 0.79138 & 0.82793 & 0.84885 \\
\hline 1581.843 & 0.70927 & 0.73273 & 0.75619 & 0.78526 & 0.82571 & 0.84584 \\
\hline 1581.963 & 0.7058 & 0.73116 & 0.75652 & 0.78631 & 0.82608 & 0.84546 \\
\hline 1582.083 & 0.69731 & 0.72489 & 0.75247 & 0.78126 & 0.82164 & 0.84208 \\
\hline 1582.203 & 0.69655 & 0.72241 & 0.74827 & 0.77953 & 0.8198 & 0.84096 \\
\hline 1582.323 & 0.68691 & 0.71434 & 0.74177 & 0.77266 & 0.81134 & 0.83348 \\
\hline 1582.443 & 0.69047 & 0.71571 & 0.74095 & 0.76961 & 0.8106 & 0.83199 \\
\hline 1582.562 & 0.68405 & 0.71078 & 0.73751 & 0.76649 & 0.80621 & 0.82901 \\
\hline 1582.682 & 0.68787 & 0.71114 & 0.73441 & 0.76533 & 0.80511 & 0.82641 \\
\hline 1582.802 & 0.67707 & 0.70431 & 0.73155 & 0.75804 & 0.79818 & 0.81863 \\
\hline 1582.922 & 0.68058 & 0.70395 & 0.72732 & 0.75616 & 0.79782 & 0.81494 \\
\hline
\end{tabular}


Table A2. Cont.

\begin{tabular}{|c|c|c|c|c|c|c|}
\hline Wavelength & NDII & NDII & NDII & NDII & NDII & NDII \\
\hline $\mathrm{nm}$ & $\mathrm{mW}$ & $\mathrm{mW}$ & $\mathrm{mW}$ & $\mathrm{mW}$ & $\mathrm{mW}$ & $\mathrm{mW}$ \\
\hline & DI Water & $25.2 \mathrm{ppm}$ & Water 2 & $43.5 \mathrm{ppm}$ & Water 3 & $64.0 \mathrm{ppm}$ \\
\hline 1583.042 & 0.66695 & 0.69543 & 0.72391 & 0.75247 & 0.79346 & 0.80942 \\
\hline 1583.162 & 0.66529 & 0.69318 & 0.72107 & 0.74915 & 0.7902 & 0.80722 \\
\hline 1583.282 & 0.65903 & 0.68993 & 0.72083 & 0.7474 & 0.7902 & 0.80649 \\
\hline 1583.402 & 0.65319 & 0.68382 & 0.71445 & 0.74397 & 0.78767 & 0.80282 \\
\hline 1583.521 & 0.6524 & 0.68149 & 0.71058 & 0.74073 & 0.78262 & 0.80026 \\
\hline 1583.641 & 0.64782 & 0.67595 & 0.70408 & 0.73456 & 0.77579 & 0.79625 \\
\hline 1583.761 & 0.64309 & 0.67123 & 0.69937 & 0.72878 & 0.77399 & 0.7908 \\
\hline 1583.881 & 0.63588 & 0.66555 & 0.69522 & 0.72337 & 0.76756 & 0.78319 \\
\hline 1584.001 & 0.63381 & 0.66165 & 0.68949 & 0.71975 & 0.76399 & 0.7803 \\
\hline 1584.121 & 0.62773 & 0.65671 & 0.68569 & 0.71568 & 0.76186 & 0.77814 \\
\hline 1584.241 & 0.61853 & 0.64836 & 0.67819 & 0.71037 & 0.7537 & 0.76916 \\
\hline 1584.361 & 0.60992 & 0.64154 & 0.67316 & 0.70439 & 0.74735 & 0.76415 \\
\hline 1584.48 & 0.60254 & 0.63362 & 0.6647 & 0.69597 & 0.73962 & 0.75845 \\
\hline 1584.6 & 0.6022 & 0.63189 & 0.66158 & 0.69422 & 0.73682 & 0.75596 \\
\hline 1584.72 & 0.59431 & 0.62592 & 0.65753 & 0.69039 & 0.73333 & 0.75135 \\
\hline 1584.84 & 0.59387 & 0.62376 & 0.65365 & 0.68749 & 0.72845 & 0.74711 \\
\hline 1584.96 & 0.58845 & 0.61765 & 0.64685 & 0.6809 & 0.72533 & 0.73972 \\
\hline 1585.08 & 0.57725 & 0.60943 & 0.64161 & 0.6729 & 0.71771 & 0.73445 \\
\hline 1585.2 & 0.57635 & 0.60687 & 0.63739 & 0.67233 & 0.71668 & 0.72921 \\
\hline 1585.32 & 0.56972 & 0.60321 & 0.6367 & 0.66935 & 0.71461 & 0.72851 \\
\hline 1585.44 & 0.56321 & 0.59888 & 0.63455 & 0.66597 & 0.71288 & 0.72607 \\
\hline 1585.559 & 0.55866 & 0.59237 & 0.62608 & 0.65811 & 0.70533 & 0.71913 \\
\hline 1585.679 & 0.55165 & 0.58613 & 0.62061 & 0.65182 & 0.69952 & 0.71255 \\
\hline 1585.799 & 0.54801 & 0.57951 & 0.61101 & 0.64668 & 0.69441 & 0.70636 \\
\hline 1585.919 & 0.54668 & 0.57817 & 0.60966 & 0.64392 & 0.69339 & 0.70567 \\
\hline 1586.039 & 0.53969 & 0.57366 & 0.60763 & 0.6433 & 0.69237 & 0.70361 \\
\hline 1586.159 & 0.53413 & 0.56668 & 0.59923 & 0.6326 & 0.68324 & 0.69642 \\
\hline 1586.279 & 0.52738 & 0.55932 & 0.59126 & 0.62641 & 0.6755 & 0.68791 \\
\hline 1586.399 & 0.52211 & 0.5515 & 0.58089 & 0.61809 & 0.6678 & 0.6781 \\
\hline 1586.518 & 0.51973 & 0.55068 & 0.58163 & 0.61975 & 0.6678 & 0.67911 \\
\hline 1586.638 & 0.50932 & 0.54503 & 0.58074 & 0.61749 & 0.66513 & 0.67844 \\
\hline 1586.758 & 0.50863 & 0.54185 & 0.57507 & 0.60999 & 0.65881 & 0.67339 \\
\hline 1586.878 & 0.49881 & 0.53291 & 0.56701 & 0.60341 & 0.65186 & 0.66401 \\
\hline 1586.998 & 0.49703 & 0.52976 & 0.56249 & 0.59789 & 0.64922 & 0.65901 \\
\hline 1587.118 & 0.4939 & 0.52645 & 0.559 & 0.59659 & 0.64363 & 0.65735 \\
\hline 1587.238 & 0.48947 & 0.52242 & 0.55537 & 0.59169 & 0.6397 & 0.65138 \\
\hline 1587.358 & 0.48467 & 0.51897 & 0.55327 & 0.58996 & 0.64035 & 0.65138 \\
\hline
\end{tabular}


Table A2. Cont.

\begin{tabular}{|c|c|c|c|c|c|c|}
\hline Wavelength & NDII & NDII & NDII & NDII & NDII & NDII \\
\hline $\mathrm{nm}$ & $\mathrm{mW}$ & $\mathrm{mW}$ & $\mathrm{mW}$ & $\mathrm{mW}$ & $\mathrm{mW}$ & $\mathrm{mW}$ \\
\hline & DI Water & $25.2 \mathrm{ppm}$ & Water 2 & $43.5 \mathrm{ppm}$ & Water 3 & $64.0 \mathrm{ppm}$ \\
\hline 1587.478 & 0.47755 & 0.51425 & 0.55095 & 0.58558 & 0.63676 & 0.64675 \\
\hline 1587.597 & 0.47758 & 0.51113 & 0.54468 & 0.58067 & 0.63186 & 0.64312 \\
\hline 1587.717 & 0.47239 & 0.50477 & 0.53715 & 0.57529 & 0.62602 & 0.63557 \\
\hline 1587.837 & 0.47051 & 0.5039 & 0.53729 & 0.5732 & 0.62342 & 0.63427 \\
\hline 1587.957 & 0.47251 & 0.50358 & 0.53465 & 0.57208 & 0.62213 & 0.631 \\
\hline 1588.077 & 0.46462 & 0.49607 & 0.52752 & 0.56647 & 0.61568 & 0.62709 \\
\hline 1588.197 & 0.45914 & 0.48922 & 0.5193 & 0.55914 & 0.60894 & 0.62059 \\
\hline 1588.317 & 0.45084 & 0.48148 & 0.51212 & 0.54905 & 0.60095 & 0.61381 \\
\hline 1588.437 & 0.45141 & 0.48015 & 0.50889 & 0.54784 & 0.59809 & 0.60995 \\
\hline 1588.556 & 0.44547 & 0.47564 & 0.50581 & 0.54353 & 0.59587 & 0.60834 \\
\hline 1588.676 & 0.43471 & 0.46712 & 0.49953 & 0.53934 & 0.58764 & 0.5997 \\
\hline 1588.796 & 0.4273 & 0.45842 & 0.48954 & 0.5309 & 0.57978 & 0.59269 \\
\hline 1588.916 & 0.42047 & 0.45245 & 0.48443 & 0.52407 & 0.5729 & 0.58478 \\
\hline 1589.036 & 0.41625 & 0.44773 & 0.47921 & 0.51927 & 0.56885 & 0.57974 \\
\hline 1589.156 & 0.41291 & 0.4437 & 0.47449 & 0.51377 & 0.56419 & 0.5766 \\
\hline 1589.276 & 0.4109 & 0.44075 & 0.4706 & 0.51116 & 0.55986 & 0.57096 \\
\hline 1589.396 & 0.40296 & 0.43365 & 0.46434 & 0.50269 & 0.554 & 0.56721 \\
\hline 1589.516 & 0.39574 & 0.42757 & 0.4594 & 0.49843 & 0.5494 & 0.56069 \\
\hline 1589.635 & 0.39227 & 0.42196 & 0.45165 & 0.48883 & 0.54389 & 0.55357 \\
\hline 1589.755 & 0.38384 & 0.4163 & 0.44876 & 0.48913 & 0.53993 & 0.55111 \\
\hline 1589.875 & 0.38351 & 0.41296 & 0.44241 & 0.48185 & 0.53567 & 0.54558 \\
\hline 1589.995 & 0.38148 & 0.41015 & 0.43882 & 0.47826 & 0.53052 & 0.54435 \\
\hline 1590.115 & 0.37601 & 0.4033 & 0.43059 & 0.47168 & 0.5251 & 0.53946 \\
\hline 1590.235 & 0.36726 & 0.39458 & 0.4219 & 0.46322 & 0.5164 & 0.53154 \\
\hline 1590.355 & 0.36501 & 0.3926 & 0.42019 & 0.46061 & 0.5152 & 0.52821 \\
\hline 1590.474 & 0.36299 & 0.3894 & 0.41581 & 0.4554 & 0.50984 & 0.52368 \\
\hline 1590.594 & 0.35732 & 0.38679 & 0.41626 & 0.45618 & 0.50924 & 0.52247 \\
\hline 1590.714 & 0.34983 & 0.3807 & 0.41157 & 0.45002 & 0.50242 & 0.51646 \\
\hline 1590.834 & 0.34006 & 0.37156 & 0.40306 & 0.44305 & 0.49329 & 0.50809 \\
\hline 1590.954 & 0.33374 & 0.36498 & 0.39622 & 0.43564 & 0.48655 & 0.50066 \\
\hline 1591.074 & 0.33253 & 0.36306 & 0.39359 & 0.43164 & 0.48363 & 0.49357 \\
\hline 1591.194 & 0.33218 & 0.36128 & 0.39038 & 0.43068 & 0.48188 & 0.49475 \\
\hline 1591.314 & 0.33136 & 0.35972 & 0.38808 & 0.42742 & 0.47695 & 0.49062 \\
\hline 1591.434 & 0.31875 & 0.35045 & 0.38215 & 0.41886 & 0.47289 & 0.48417 \\
\hline 1591.553 & 0.31471 & 0.34314 & 0.37157 & 0.41003 & 0.46196 & 0.47427 \\
\hline 1591.673 & 0.31183 & 0.34006 & 0.36829 & 0.40951 & 0.45967 & 0.47166 \\
\hline 1591.793 & 0.30852 & 0.33671 & 0.3649 & 0.40493 & 0.45596 & 0.46674 \\
\hline
\end{tabular}


Table A2. Cont.

\begin{tabular}{|c|c|c|c|c|c|c|}
\hline Wavelength & NDII & NDII & NDII & NDII & NDII & NDII \\
\hline $\mathrm{nm}$ & $\mathrm{mW}$ & $\mathrm{mW}$ & $\mathrm{mW}$ & $\mathrm{mW}$ & $\mathrm{mW}$ & $\mathrm{mW}$ \\
\hline & DI Water & $25.2 \mathrm{ppm}$ & Water 2 & 43.5 ppm & Water 3 & $64.0 \mathrm{ppm}$ \\
\hline 1591.913 & 0.30491 & 0.33337 & 0.36183 & 0.39944 & 0.4534 & 0.4653 \\
\hline 1592.033 & 0.29943 & 0.32782 & 0.35621 & 0.39359 & 0.44489 & 0.46041 \\
\hline 1592.153 & 0.29143 & 0.31886 & 0.34629 & 0.38429 & 0.4387 & 0.4544 \\
\hline 1592.273 & 0.29393 & 0.31749 & 0.34105 & 0.3799 & 0.43449 & 0.44927 \\
\hline 1592.393 & 0.29109 & 0.31585 & 0.34061 & 0.38286 & 0.43421 & 0.4487 \\
\hline 1592.512 & 0.2936 & 0.31797 & 0.34234 & 0.38004 & 0.43281 & 0.44728 \\
\hline 1592.632 & 0.28931 & 0.31352 & 0.33773 & 0.37727 & 0.42723 & 0.44331 \\
\hline 1592.752 & 0.28369 & 0.30704 & 0.33039 & 0.36993 & 0.42361 & 0.43597 \\
\hline 1592.872 & 0.27234 & 0.29884 & 0.32534 & 0.36313 & 0.41476 & 0.42783 \\
\hline 1592.992 & 0.2732 & 0.29851 & 0.32382 & 0.36109 & 0.41283 & 0.42449 \\
\hline 1593.112 & 0.26826 & 0.29601 & 0.32376 & 0.36004 & 0.41091 & 0.42142 \\
\hline 1593.232 & 0.26865 & 0.2946 & 0.32055 & 0.35601 & 0.40734 & 0.42059 \\
\hline 1593.352 & 0.26116 & 0.28802 & 0.31488 & 0.35141 & 0.40133 & 0.41504 \\
\hline 1593.472 & 0.25245 & 0.27863 & 0.30481 & 0.34292 & 0.39346 & 0.40842 \\
\hline 1593.591 & 0.24561 & 0.27393 & 0.30225 & 0.33717 & 0.38887 & 0.40294 \\
\hline 1593.711 & 0.24993 & 0.27327 & 0.29661 & 0.33406 & 0.38376 & 0.3972 \\
\hline 1593.831 & 0.24602 & 0.26951 & 0.293 & 0.32905 & 0.37974 & 0.39529 \\
\hline 1593.951 & 0.23659 & 0.26144 & 0.28629 & 0.3217 & 0.37068 & 0.38959 \\
\hline 1594.071 & 0.23407 & 0.25492 & 0.27577 & 0.3136 & 0.363 & 0.37962 \\
\hline 1594.191 & 0.22427 & 0.24683 & 0.26939 & 0.3047 & 0.35538 & 0.37213 \\
\hline 1594.311 & 0.22383 & 0.2458 & 0.26777 & 0.29849 & 0.3512 & 0.3692 \\
\hline 1594.431 & 0.21726 & 0.23943 & 0.2616 & 0.29888 & 0.34444 & 0.36442 \\
\hline 1594.55 & 0.21433 & 0.23413 & 0.25393 & 0.29086 & 0.34055 & 0.35781 \\
\hline 1594.67 & 0.21065 & 0.22993 & 0.24921 & 0.28643 & 0.33643 & 0.35334 \\
\hline 1594.79 & 0.20236 & 0.22258 & 0.2428 & 0.2801 & 0.32566 & 0.34472 \\
\hline 1594.91 & 0.20005 & 0.21892 & 0.23779 & 0.27559 & 0.32311 & 0.34134 \\
\hline 1595.03 & 0.19201 & 0.21428 & 0.23655 & 0.27449 & 0.31804 & 0.33693 \\
\hline 1595.15 & 0.18687 & 0.21115 & 0.23543 & 0.26966 & 0.31551 & 0.33409 \\
\hline 1595.27 & 0.18641 & 0.20815 & 0.22989 & 0.26477 & 0.30946 & 0.32869 \\
\hline 1595.39 & 0.17714 & 0.19984 & 0.22254 & 0.25745 & 0.30519 & 0.32433 \\
\hline 1595.51 & 0.17003 & 0.19374 & 0.21745 & 0.25142 & 0.29746 & 0.31643 \\
\hline 1595.629 & 0.16737 & 0.18963 & 0.21189 & 0.24534 & 0.29374 & 0.3091 \\
\hline 1595.749 & 0.17025 & 0.18981 & 0.20937 & 0.24337 & 0.28929 & 0.30733 \\
\hline 1595.869 & 0.16465 & 0.18559 & 0.20653 & 0.23945 & 0.28757 & 0.30557 \\
\hline 1595.989 & 0.15777 & 0.17975 & 0.20173 & 0.23421 & 0.27971 & 0.30181 \\
\hline 1596.109 & 0.15917 & 0.17733 & 0.19549 & 0.22814 & 0.27362 & 0.29556 \\
\hline 1596.229 & 0.15205 & 0.17075 & 0.18945 & 0.22328 & 0.26708 & 0.28737 \\
\hline
\end{tabular}


Table A2. Cont.

\begin{tabular}{|c|c|c|c|c|c|c|}
\hline Wavelength & NDII & NDII & NDII & NDII & NDII & NDII \\
\hline $\mathrm{nm}$ & $\mathrm{mW}$ & $\mathrm{mW}$ & $\mathrm{mW}$ & $\mathrm{mW}$ & $\mathrm{mW}$ & $\mathrm{mW}$ \\
\hline & DI Water & $25.2 \mathrm{ppm}$ & Water 2 & $43.5 \mathrm{ppm}$ & Water 3 & $64.0 \mathrm{ppm}$ \\
\hline 1596.349 & 0.1483 & 0.16799 & 0.18768 & 0.22051 & 0.2649 & 0.28416 \\
\hline 1596.469 & 0.15142 & 0.17009 & 0.18876 & 0.21971 & 0.26515 & 0.2817 \\
\hline 1596.588 & 0.14725 & 0.16559 & 0.18393 & 0.21453 & 0.25961 & 0.27851 \\
\hline 1596.708 & 0.1398 & 0.15856 & 0.17732 & 0.20968 & 0.25221 & 0.27167 \\
\hline 1596.828 & 0.13635 & 0.15387 & 0.17139 & 0.20313 & 0.24603 & 0.2656 \\
\hline 1596.948 & 0.13061 & 0.15068 & 0.17075 & 0.20191 & 0.24461 & 0.26245 \\
\hline 1597.068 & 0.13024 & 0.14968 & 0.16912 & 0.20022 & 0.24391 & 0.26149 \\
\hline 1597.188 & 0.13361 & 0.15015 & 0.16669 & 0.19991 & 0.24037 & 0.25836 \\
\hline 1597.308 & 0.12101 & 0.14322 & 0.16543 & 0.19396 & 0.23497 & 0.25523 \\
\hline 1597.428 & 0.123 & 0.14029 & 0.15758 & 0.18968 & 0.23123 & 0.24854 \\
\hline 1597.547 & 0.11168 & 0.13325 & 0.15482 & 0.18275 & 0.22588 & 0.24118 \\
\hline 1597.667 & 0.11353 & 0.13169 & 0.14985 & 0.17821 & 0.22101 & 0.23858 \\
\hline 1597.787 & 0.10959 & 0.12799 & 0.14639 & 0.17606 & 0.21802 & 0.23434 \\
\hline 1597.907 & 0.11296 & 0.12759 & 0.14222 & 0.17098 & 0.21526 & 0.22989 \\
\hline 1598.027 & 0.10872 & 0.12511 & 0.1415 & 0.16767 & 0.20908 & 0.22779 \\
\hline 1598.147 & 0.10307 & 0.11835 & 0.13363 & 0.16096 & 0.20452 & 0.22057 \\
\hline 1598.267 & 0.09768 & 0.1139 & 0.13012 & 0.15318 & 0.19796 & 0.21387 \\
\hline 1598.387 & 0.09588 & 0.11242 & 0.12896 & 0.15435 & 0.19615 & 0.21526 \\
\hline 1598.506 & 0.0978 & 0.11447 & 0.13114 & 0.15487 & 0.19638 & 0.21364 \\
\hline 1598.626 & 0.09874 & 0.11418 & 0.12962 & 0.15431 & 0.19368 & 0.21203 \\
\hline 1598.746 & 0.09054 & 0.10727 & 0.124 & 0.14413 & 0.18585 & 0.20243 \\
\hline 1598.866 & 0.09031 & 0.10349 & 0.11667 & 0.14142 & 0.17896 & 0.19698 \\
\hline 1598.986 & 0.08617 & 0.10107 & 0.11597 & 0.14209 & 0.17786 & 0.19291 \\
\hline 1599.106 & 0.08495 & 0.10214 & 0.11933 & 0.1412 & 0.17543 & 0.19359 \\
\hline 1599.226 & 0.08423 & 0.0999 & 0.11557 & 0.13917 & 0.17323 & 0.19133 \\
\hline 1599.346 & 0.08195 & 0.09659 & 0.11123 & 0.136 & 0.17081 & 0.18729 \\
\hline 1599.466 & 0.07659 & 0.09163 & 0.10667 & 0.12682 & 0.16208 & 0.18237 \\
\hline 1599.585 & 0.07036 & 0.08698 & 0.1036 & 0.12351 & 0.16099 & 0.17726 \\
\hline 1599.705 & 0.07307 & 0.08698 & 0.10089 & 0.12151 & 0.15601 & 0.1746 \\
\hline 1599.825 & 0.07467 & 0.08864 & 0.10261 & 0.12187 & 0.15774 & 0.17526 \\
\hline 1599.945 & 0.07456 & 0.0862 & 0.09784 & 0.11894 & 0.15213 & 0.17018 \\
\hline 1600.065 & 0.06658 & 0.08091 & 0.09524 & 0.11376 & 0.15062 & 0.16535 \\
\hline 1600.185 & 0.06177 & 0.07609 & 0.09041 & 0.10599 & 0.14293 & 0.16011 \\
\hline 1600.305 & 0.06521 & 0.07565 & 0.08609 & 0.10503 & 0.13762 & 0.15924 \\
\hline 1600.425 & 0.06803 & 0.0791 & 0.09017 & 0.1076 & 0.13741 & 0.1575 \\
\hline 1600.544 & 0.06521 & 0.07713 & 0.08905 & 0.10692 & 0.13698 & 0.1575 \\
\hline 1600.664 & 0.0621 & 0.07412 & 0.08614 & 0.10265 & 0.13192 & 0.15533 \\
\hline
\end{tabular}


Table A2. Cont.

\begin{tabular}{|c|c|c|c|c|c|c|}
\hline Wavelength & NDII & NDII & NDII & NDII & NDII & NDII \\
\hline $\mathrm{nm}$ & $\mathbf{m W}$ & $\mathbf{m W}$ & $\mathbf{m W}$ & $\mathrm{mW}$ & $\mathrm{mW}$ & $\mathbf{m W}$ \\
\hline & DI Water & $25.2 \mathrm{ppm}$ & Water 2 & $43.5 \mathrm{ppm}$ & Water 3 & $64.0 \mathrm{ppm}$ \\
\hline 1600.784 & 0.06797 & 0.07139 & 0.07481 & 0.09294 & 0.12563 & 0.14712 \\
\hline 1600.904 & 0.0595 & 0.06857 & 0.07764 & 0.09266 & 0.12354 & 0.14326 \\
\hline 1601.024 & 0.05365 & 0.06397 & 0.07429 & 0.09003 & 0.12146 & 0.13559 \\
\hline 1601.144 & 0.06275 & 0.06797 & 0.07319 & 0.09182 & 0.12125 & 0.13963 \\
\hline 1601.264 & 0.05572 & 0.06407 & 0.07242 & 0.08996 & 0.11876 & 0.13517 \\
\hline 1601.384 & 0.05036 & 0.05735 & 0.06434 & 0.08107 & 0.1105 & 0.12609 \\
\hline 1601.504 & 0.03907 & 0.05078 & 0.06249 & 0.07668 & 0.10415 & 0.12399 \\
\hline 1601.623 & 0.03699 & 0.04642 & 0.05585 & 0.07262 & 0.09744 & 0.12064 \\
\hline 1601.743 & 0.04603 & 0.05094 & 0.05585 & 0.06936 & 0.09744 & 0.12043 \\
\hline 1601.863 & 0.03358 & 0.04483 & 0.05608 & 0.06834 & 0.09906 & 0.11606 \\
\hline 1601.983 & 0.03574 & 0.04404 & 0.05234 & 0.06646 & 0.09542 & 0.11233 \\
\hline 1602.103 & 0.03452 & 0.03982 & 0.04512 & 0.06017 & 0.08717 & 0.10758 \\
\hline 1602.223 & 0.03154 & 0.0362 & 0.04086 & 0.05541 & 0.08278 & 0.09878 \\
\hline 1602.343 & 0.01942 & 0.03176 & 0.0441 & 0.05356 & 0.08059 & 0.10021 \\
\hline 1602.463 & 0.03481 & 0.03756 & 0.04031 & 0.05504 & 0.07921 & 0.09898 \\
\hline 1602.582 & 0.02745 & 0.03395 & 0.04045 & 0.05147 & 0.07624 & 0.09248 \\
\hline 1602.702 & 0.02854 & 0.03155 & 0.03456 & 0.05134 & 0.06954 & 0.09026 \\
\hline 1602.822 & 0.0235 & 0.02832 & 0.03314 & 0.04323 & 0.06778 & 0.08423 \\
\hline 1602.942 & 0.0235 & 0.02832 & 0.03314 & 0.04456 & 0.067 & 0.08323 \\
\hline 1603.062 & 0.02442 & 0.03009 & 0.03576 & 0.04709 & 0.0668 & 0.07943 \\
\hline 1603.182 & 0.02383 & 0.0278 & 0.03177 & 0.0439 & 0.06388 & 0.08003 \\
\hline 1603.302 & 0.02276 & 0.02485 & 0.02694 & 0.04363 & 0.0598 & 0.07645 \\
\hline 1603.422 & 0.01307 & 0.01799 & 0.02291 & 0.03497 & 0.05535 & 0.07149 \\
\hline 1603.542 & 0.0225 & 0.0202 & 0.0179 & 0.02864 & 0.05304 & 0.06618 \\
\hline 1603.661 & 0.01551 & 0.01702 & 0.01853 & 0.02854 & 0.04882 & 0.05992 \\
\hline 1603.781 & 0.01964 & 0.0201 & 0.02056 & 0.0305 & 0.05208 & 0.0607 \\
\hline 1603.901 & 0.00754 & 0.01328 & 0.01902 & 0.0306 & 0.0492 & 0.06226 \\
\hline 1604.021 & 0.01217 & 0.01344 & 0.01471 & 0.02463 & 0.04672 & 0.05236 \\
\hline 1604.141 & 0.00703 & 0.01022 & 0.01341 & 0.0197 & 0.03912 & 0.05255 \\
\hline 1604.261 & $5.90 \times 10^{-4}$ & 0.00738 & 0.01417 & 0.01474 & 0.04158 & 0.05081 \\
\hline 1604.381 & 0.00491 & 0.01109 & 0.01727 & 0.01706 & 0.03855 & 0.05274 \\
\hline 1604.5 & 0.00188 & 0.00814 & 0.0144 & 0.01796 & 0.03893 & 0.04908 \\
\hline 1604.62 & 0.00543 & 0.00819 & 0.01095 & 0.01793 & 0.03912 & 0.0439 \\
\hline
\end{tabular}




\section{References}

1. Akbarnezhad, S.; Amini, A.; Goharrizi, A.S.; Rainey, T.; Morawska, L. Capacity of quartz fibers with high filtration efficiency for capturing soot aerosol particles. Int. J. Environ. Sci. Technol. 2018, 15, 1039-1048. [CrossRef]

2. Shi, R.; Huang, C.; Zhang, L.; Amini, A.; Liu, K.; Shi, Y.; Bao, S.; Wang, N.; Cheng, C. Three Dimensional Sculpturing of Vertical Nanowire Arrays by Conventional Photolithography. Sci. Rep. 2016, 6, 18886. [CrossRef] [PubMed]

3. Masindi, V.; Muedi, K.L. Environmental Contamination by Heavy Metals; IntechOpen: London, UK, 2018; p. 7; [CrossRef]

4. Goel, A.D.; Chowgule, R.V. Outbreak investigation of lead neurotoxicity in children from artificial jewelry cottage industry. Environ. Health Prev. Med. 2019, 24, 30. [CrossRef] [PubMed]

5. Docherty, B.; Kariuki, M. Creating an Effective Corrosion Control Program to Eliminate Lead in Drinking Water in Hamilton, Ontario. J. AWWA 2019, 111, 28-38. [CrossRef]

6. Zahran, S.; McElmurry, S.P.; Sadler, R.C. Four phases of the Flint Water Crisis: Evidence from blood lead levels in children. Environ. Res. 2017, 157, 160-172. [CrossRef]

7. Delile, H.; Blichert-Toft, J.; Goiran, J.P.; Keay, S.; Albarède, F. Lead in ancient Rome's city waters. Proc. Natl. Acad. Sci. USA 2014, 111, 6594-6599. [CrossRef]

8. DeSantis, M.K.; Triantafyllidou, S.; Schock, M.R.; Lytle, D.A. Mineralogical Evidence of Galvanic Corrosion in Drinking Water Lead Pipe Joints. Environ. Sci. Technol. 2018, 52, 3365-3374. [CrossRef]

9. Mayans, L. Lead Poisoning in Children. Am. Fam. Physician 2019, 100, 24-30.

10. Cantor, A.G.; Hendrickson, R.; Blazina, I. Screening for Elevated Blood Lead Levels in Children and Pregnant Women: US Preventive Services Task Force Recommendation Statement. JAMA 2019, 321, 1502-1509. [CrossRef]

11. Ettinger, A.S.; Egan, K.B.; Homa, D.M.; Brown, M.J. Blood Lead Levels in U.S. Women of Childbearing Age, 1976-2016. Environ. Health Perspect. 2020, 128, 17012. [CrossRef]

12. Paul, S.; Mandal, A.; Bhattacharjee, P.; Chakraborty, S.; Paul, R.; Kumar Mukhopadhyay, B. Evaluation of water quality and toxicity after exposure of lead nitrate in fresh water fish, major source of water pollution. Egypt. J. Aquat. Res. 2019, 45, 345-351. [CrossRef]

13. Mason, L.H.; Harp, J.P.; Han, D.Y. Pb neurotoxicity: Neuropsychological effects of lead toxicity. Biomed. Res. Int. 2014, 2014, 840547. [CrossRef]

14. Cai, S.Z.; Zhao, L.N.; Liu, J.; Ji, Y.T.; Shi, X.Y.; Ma, Z.R.; Lv, X.H.; Chen, K.; Chen, Y. Allicin alleviates lead-induced hematopoietic stem cell aging by up-regulating PKM2. Biosci. Rep. 2019, 39, BSR20190243. [CrossRef]

15. Radulescu, A.; Lundgren, S. A pharmacokinetic model of lead absorption and calcium competitive dynamics. Sci. Rep. 2019, 9, 14225. [CrossRef]

16. de Mattos, G.F.; Costa, C.; Savio, F.; Alonso, M.; Nicolson, G.L. Lead poisoning: acute exposure of the heart to lead ions promotes changes in cardiac function and Cav1.2 ion channels. Biophys. Rev. 2017, 9, 807-825. [CrossRef]

17. Rajendran, S. Lead Toxicity on Male Reproductive System and its Mechanism: A Review. Res. J. Pharm. Technol. 2018, 11, 1228-1232. [CrossRef]

18. Kumar, S. Occupational and Environmental Exposure to Lead and Reproductive Health Impairment: An Overview. Indian J. Occup. Environ. Med. 2018, 22, 128-137. [CrossRef]

19. Vu, H.H.; Gu, S.; Thriveni, T.; Khan, D.M.; Tuan, Q.L.; Ahn, W.J. Sustainable Treatment for Sulfate and Lead Removal from Battery Wastewater. Sustainability 2019, 11, 3497. [CrossRef]

20. Zeng, C.; Hu, H.; Feng, X.; Wang, K.; Zhang, Q. Activating $\mathrm{CaCO}_{3}$ to enhance lead removal from lead-zinc solution to serve as green technology for the purification of mine tailings. Chemosphere 2020, 249, 126227. [CrossRef]

21. Tang, X.; Wang, P.Y.; Buchter, G. Ion-Selective Electrodes for Detection of Lead (II) in Drinking Water: A Mini-Review. Environments 2018, 5, 95. [CrossRef]

22. Nguyen, H.; Sung, Y.; O'Shaughnessy, K.; Shan, X.; Shih, W.C. Smartphone Nanocolorimetry for On-Demand Lead Detection and Quantitation in Drinking Water. Anal. Chem. 2018, 90, 11517-11522. [CrossRef]

23. Zhang, Z.; Ma, P.; Li, J.; Sun, Y.; Shi, H.; Chen, N.; Zhang, X.; Chen, H. Colorimetric and SERS dual-mode detection of lead Ions based on Au-Ag core-shell nanospheres: featuring quick screening with ultra-high sensitivity. Opt. Express 2019, 27, 29248-29260. [CrossRef]

24. Seitz, H.; Stahl, F.; Walter, J.G. Catalytically Active Nucleic Acids; Advances in Biochemical Engineering/Biotechnology; Springer International Publishing: Berlin/Heidelberg, Germany, 2019.

25. Lin, W.C.; Li, Z.; Burns, M.A. A Drinking Water Sensor for Lead and Other Heavy Metals. Anal. Chem. 2017, 89, 8748-8756. [CrossRef]

26. Kanellis, V.G. Sensitivity limits of biosensors used for the detection of metals in drinking water. Biophys. Rev. 2018, 10, 1415-1426. [CrossRef]

27. Yu, C.; Shao, Z.; Hou, H. A functionalized metal-organic framework decorated with O- groups showing excellent performance for lead(ii) removal from aqueous solution. Chem. Sci. 2017, 8, 7611-7619. [CrossRef]

28. Fang, X.; Zong, B.; Mao, S. Metal-Organic Framework-Based Sensors for Environmental Contaminant Sensing. Nano-Micro Lett. 2018, 10, 64. [CrossRef] 
29. Okoro, H.K.; Ayika, S.O.; Ngila, J.C.; Tella, A.C. Rising profile on the use of metal-organic frameworks (MOFs) for the removal of heavy metals from the environment: an overview. Appl. Water Sci. 2018, 8, 169. [CrossRef]

30. Kobielska, P.A.; Howarth, A.J.; Farha, O.K.; Nayak, S. Metal-organic frameworks for heavy metal removal from water. Coord. Chem. Rev. 2018, 358, 92-107. [CrossRef]

31. Li, W.T.; Hu, Z.J.; Meng, J.; Zhang, X.; Gao, W.; Chen, M.L.; Wang, J.H. Zn-based metal organic framework-covalent organic framework composites for trace lead extraction and fluorescence detection of TNP. J. Hazard. Mater. 2021, 411, 125021. [CrossRef]

32. Tang, J.; Chen, Y.; Zhao, M.; Wang, S.; Zhang, L. Phenylthiosemicarbazide-functionalized UiO-66-NH2 as highly efficient adsorbent for the selective removal of lead from aqueous solutions. J. Hazard. Mater. 2021, 413, 125278. [CrossRef]

33. Zhao, F.; Yang, W.; Han, Y.; Luo, X.; Tang, W.; Yue, T.; Li, Z. A straightforward strategy to synthesize supramolecular amorphous zirconium metal-organic gel for efficient $\mathrm{Pb}$ (II) removal. Chem. Eng. J. 2021, 407, 126744. [CrossRef]

34. Wang, C.; Liu, X.; Keser Demir, N.; Chen, J.P.; Li, K. Applications of water stable metal-organic frameworks. Chem. Soc. Rev. 2016, 45,5107-5134. [CrossRef] [PubMed]

35. Nazari, M.; Forouzandeh, M.A.; Divarathne, C.M.; Sidiroglou, F.; Martinez, M.R.; Konstas, K.; Muir, B.W.; Hill, A.J.; Duke, M.C.; Hill, M.R.; et al. UiO-66 MOF end-face-coated optical fiber in aqueous contaminant detection. Opt. Lett. 2016, 41, 1696-1699. [CrossRef] [PubMed]

36. Nazari, M.; Amini, A.; Hill, M.R.; Cheng, C.; Samali, B. Physical and chemical reaction sensing in a mixed aqueous solution via metal-organic framework thin-film coated optical fiber. Microw. Opt. Technol. Lett. 2020, 62, 72-77. [CrossRef]

37. Nazari, M.; Rubio-Martinez, M.; Babarao, R.; Younis, A.A.; Collins, S.F.; Hill, M.R.; Duke, M.C. Aqueous contaminant detection via UiO-66 thin film optical fiber sensor platform with fast Fourier transform based spectrum analysis. J. Phys. Appl. Phys. 2017, 51, 25601. [CrossRef]

38. Vaughan, G.M. The Fabry-Perot Interferometer: History, Theory, Practice and Applications; CRC Press: Boca Raton, FL, USA, 2017; p. 604 .

39. The Mathworks, Inc. MATLAB Version 9.5.0.955555 (R2018b); The Mathworks, Inc.: Natick, MA, USA, 2018.

40. Khayyun, T.S.; Mseer, A.H. Comparison of the experimental results with the Langmuir and Freundlich models for copper removal on limestone adsorbent. Appl. Water Sci. 2019, 9, 170. [CrossRef]

41. Ricco, R.; Konstas, K.; Styles, M.J.; Richardson, J.J.; Babarao, R.; Suzuki, K.; Scopece, P.; Falcaro, P. Lead(ii) uptake by aluminium based magnetic framework composites (MFCs) in water. J. Mater. Chem. 2015, 3, 19822-19831. [CrossRef]

42. Dignam, T.; Kaufmann, R.B.; LeStourgeon, L.; Mary, J.S. Control of Lead Sources in the United States, 1970-2017: Public Health Progress and Current Challenges to Eliminating Lead Exposure. J. Public Health Manag. Pract. 2019, 25, S13-S22. [CrossRef]

43. Evano, N.; Abdi, R.; Poulain, M. Lifetime modeling of silica optical fiber in static fatigue test. J. Appl. Res. Technol. 2016, 14, 278-285. [CrossRef]

44. Sun, Y.; Sun, Q.; Huang, H.; Aguila, B.; Niu, Z.; Perman, J.A.; Ma, S. A molecular-level superhydrophobic external surface to improve the stability of metal-organic frameworks. J. Mater. Chem. 2017, 5, 18770-18776. [CrossRef]

45. Nazari, M.; Rubio-Martinez, M.; Tobias, G.; Barrio, J.P.; Babarao, R.; Nazari, F.; Konstas, K.; Muir, B.W.; Collins, S.F.; Hill, A.J.; et al. Metal-Organic-Framework-Coated Optical Fibers as Light-Triggered Drug Delivery Vehicles. Adv. Funct. Mater. 2016, 26, 3244-3249. [CrossRef]

46. Krol, A.; Mizerna, K.; Bozym, M. An assessment of pH-dependent release and mobility of heavy metals from metallurgical slag. J. Hazard. Mater. 2020, 384, 121502. [CrossRef]

47. Zhu, H.; Yuan, J.; Tan, X.; Zhang, W.; Fang, M.; Wang, X. Efficient removal of $\mathrm{Pb}^{2+}$ by Tb-MOFs: Identifying the adsorption mechanism through experimental and theoretical investigations. Environ. Sci. Nano 2019, 6, 261-272. [CrossRef]

48. Geisse, A.R.; Ngule, C.M.; Genna, D.T. Removal of lead ions from water using thiophene-functionalized metal-organic frameworks. Chem. Commun. 2020, 56, 237-240. [CrossRef]

49. Allahdin, O.; Mabingui, J.; Wartel, M.; Boughriet, A. Removal of $\mathrm{Pb}^{2+}$ ions from aqueous solutions by fixed-BED column using a modified brick: Micro structural, electrokinetic and mechanistic aspects. Appl. Clay Sci. 2017, 148, 56-67. [CrossRef]

50. Powell, K.; Brown, P.; Byrne, R.; Gajda, T.; Hefter, G.; Leuz, A.K.; Sjoberg, S.; Wanner, H. Chemical speciation of environmentally significant metals with inorganic ligands. Part 3: IUPAC Technical Report. Pure Appl. Chem. 2009, 81, 2425-2476. [CrossRef] 